\title{
Chaotic Dynamics of an Extended Duffing Oscillator Under Periodic Excitation
}

\author{
Hervé Lucas Koudahoun ${ }^{1,}$ *, Yélomè Judicaël Fernando Kpomahou ${ }^{2}$, Jean Akande ${ }^{1}$, \\ Damien Kêgnidé Kolawolé Adjaï ${ }^{1}$ \\ ${ }^{1}$ Department of Physics, University of Abomey-Calavi, Abomey-Calavi, Benin \\ ${ }^{2}$ Department of Industrial and Technical Sciences, University of Abomey, Abomey, Benin
}

Email address:

herluckou@gmail.com (H. L. Koudahoun), fkpomahou@gmail.com (Y. J. F. Kpomahou), jeanakande7@gmail.com (J. Akande), adjkolakegni@gmail.com (D. K. K. Adjaï)

${ }^{*}$ Corresponding author

\section{To cite this article:}

Hervé Lucas Koudahoun, Yélomè Judicaël Fernando Kpomahou, Jean Akande, Damien Kêgnidé Kolawolé Adjaï. Chaotic Dynamics of an Extended Duffing Oscillator Under Periodic Excitation. World Journal of Applied Physics. Vol. 3, No. 2, 2018, pp. 34-50. doi: 10.11648 j.wjap.20180302.13

Received: June 29, 2018; Accepted: July 12, 2018; Published: August 6, 2018

\begin{abstract}
In this paper, chaotic dynamics of a cubic-quintic-septic Duffing oscillator subjected to periodic excitation is investigated. The multiple scales method is used to determine the various resonance states of the model. It is found that the considered model posses thirteen resonance states whose seven are thoroughly studied. The steady-state solutions and theirs stabilities are determined. The frequency-amplitude curves show that the considered system presents mixed behavior, limit cycles, hysteresis, jump and bifurcation phenomena. It is also noticed that these phenomena are strongly influenced by quintic-septic nonlinearity and excitation amplitude. Bifurcation structures displayed by the model for each considered type of resonant states are investigated numerically using the fourth-order Runge-Kutta algorithm. As results, the quintic-septic nonlinearity, linear dissipation and excitation amplitude can be used to control the chaotic behavior of the system.
\end{abstract}

Keywords: Extended Duffing Oscillator, Resonance States, Stability, Limit Cycles, Bifurcation and Jump Phenomena, Periodic and Quasi-periodic Oscillations, Chaos

\section{Introduction}

In recent decades, the studies on chaotic dynamics of nonlinear dissipative systems have received considerable attention of many investigators in order to understand the complex dynamical behavior of these systems. These studies are of great importance because chaos has large practical applications in sciences but also in engineering and in technology. For instance chaos has been used in physics for refining the understanding of planetary orbits and also for forecasting the intensity of solar activity [1]. In medicine, biology and physiology, the chaotic behavior has been used to detect and explain some physiological phenomena [2-4]. In meteorology, chaos can be used to indicate situations of better or worse predictability. In nanotechnology, the width of the conductance correlation function is determined by the average lifetime of transient chaos. Moreover in this discipline, chaos has been used to make the channels of a few hundred microns in cross-section [5]. In communication, chaos has been used to transmit information [6]. In engineering, chaotic dynamics was used for controlling the temperature of kerosene fan heaters and the direction of air flow of air conditioners to provide useful fluctuations that are expected to be comfortable to humans [7-8], etc. In view of the existence and importance of chaos in various branches of sciences and engineering, quantitative or qualitative analysis of chaotic systems is become a challenge for investigators. There are some numerical tools such as bifurcation diagram, phase portrait, Poincaré section map, Lyapunov exponent diagram and so on that are used to measure, detect, predict or quantify the chaotic behavior of dynamical systems. Many investigators in the study of chaotic dynamics have used Lyapunov exponent as a good indicator of chaos. According to this tool, a positive value of Lyapunov exponent is a 
quantitative measure of chaos [9]. Frankly speaking, among the three fundamental forced oscillators such as Van der Pol, Rayleigh and Duffing oscillators, this latter with $\varphi^{4}$ potential having hardening or softening nonlinearity

$$
u^{\prime \prime}+\mu u^{\prime}+\left(a-b u^{2}\right) u=f \cos (\Omega \tau)
$$

where the prime (')represents a differentiation with respect to time, has been intensively used in context of various physical and engineering problems due to it rich variety of nonlinear dynamical behavior such as hysteresis, bifurcation, regular and chaotic behaviors and its potential applications [10-17]. Recently again it has been shown that Duffing oscillator with $\varphi^{6}$ potential exhibits for appropriate parameter choices a wide rich variety of dynamical behavior that the dynamical system described by equation (1) cannot display [18-19]. Furthermore it has been also shown that the quintic term of $\varphi^{6}$ Duffing oscillator is responsible to produce the hardening-softening or softening- hardening behavior so-called mixed behavior. The authors of this paper have underlined that this behavior highly unstable, can be advantageous or disadvantageous according to the type of applications [20, 21, 22]. In the light of all the above, forced damped Duffing oscillator remains up to now a very important nonlinear model to examine in the study of chaotic dynamics in nonlinear dissipative systems. Therefore we consider in this work the following equation:

$$
u^{\prime \prime}+\mu u^{\prime}+\left(1-\frac{1}{2} u^{2}\right)\left(1+\frac{\alpha}{4} u^{4}\right) u=F \cos (\Omega \tau)
$$

The particularity of this model is that it contains one parameter controlling the quintic and septic nonlinearities simultaneously. This model can arise in the modeling of a classical particle in a double-well potential [23]. It was also used to model the dynamic behavior of a cargo system [24]. It is interesting to point out that this model without damping has been obtained in the modeling of the purely elastic structures whose the tension was related to the normal strain by a power law [25]. The equation (2) is an extended form of cubic damped Duffing oscillator defined by (1). From this equation, when $\alpha=0$, the softening Duffing oscillator with $\varphi^{4}$ potential describes by equation (1) is obtained with $a=1$ and $b=\frac{1}{2}$.

As foregoing mentioned, various studies have been dedicated to the softening Duffing oscillator. The interest in this system lies in the variety of physical phenomena that it models, such as the rolling motion of a ship [26-27] and the fact that it is isomorphic with other systems of importance in physics and engineering [28]. The present study is motivated by the large variety of dynamical behavior of a softening Duffing oscillator [12] and also by the fact that the chaotic dynamics of a high-order Duffing oscillator have not been intensively studied up to now [18]. Therefore, in this work the following question is necessary to be asked in order to understand the chaotic behavior of dynamical system governed by (2): is that the parameter $\alpha$ controlling the quintic and septic nonlinearities simultaneously can affect the dynamics of softening Duffing oscillator equation? In order to answer to this equation, the authors of this paper suppose that $\alpha$ has significant effect on the dynamics of the softening Duffing oscillator with $\varphi^{4}$ potential. To verify this assumption, we determine the various resonant states of (2) by means of the multiple scales method as well as the influence of the significant parameters on the each steady-state solution (Section 2). We afterward analyze its bifurcation and transition to chaos using numerical simulations (Section 3). Finally we present the conclusion of this work (Section 4).

\section{Resonance States}

In this section, we use the multiple scales method [29] for investigating the various resonance states of (2) as well as the effects of the significant parameters on the each steady-state response. To apply this method, we introduce into equation (2) the small perturbation parameter $\varepsilon$ such as $0 \leq \varepsilon<1$. Thus, equation (2) can be written as follows

$$
u^{\prime \prime}+u=\varepsilon\left(-\mu u^{\prime}+\frac{1}{2} u^{3}-\frac{\alpha}{4}\left(1-\frac{1}{2} u^{2}\right) u^{5}\right)+F \cos (\Omega \tau)
$$

where $\mu=\varepsilon \mu$ and $\alpha=\varepsilon \alpha$. Now, we seek an uniform first-order solution of equation (3)in the form:

$$
u(\tau, \varepsilon)=u_{0}\left(T_{0}, T_{1}\right)+\varepsilon u_{1}\left(T_{0}, T_{1}\right)+0\left(\varepsilon^{2}\right)
$$

where $T_{o}=\tau$ and $T_{1}=\varepsilon \tau$ represent the fast and slow time scales respectively. In terms of the new time scales, the time derivatives become

$$
\frac{d}{d \tau}=D_{0}+\varepsilon D_{1}+0\left(\varepsilon^{2}\right) \& \frac{d^{2}}{d \tau^{2}}=D_{0}^{2}+2 \varepsilon D_{0} D_{1}+0\left(\varepsilon^{2}\right)
$$

Where $D_{n}=\frac{\partial}{\partial T_{n}}, n=0,1$ represents differential operator.

\subsection{Primary Resonant State}

In this part, we put that $F=\varepsilon F$. The relationship between both natural and external frequencies is given by $\omega=1+\varepsilon \sigma$, where $\sigma$ is the detuning parameter. Inserting equations (4) and (5) into (3) and equating the coefficients of like power of zero-order $\varepsilon^{0}$ and first-order $\varepsilon^{1}$ on both sides, we obtain:

$$
\begin{gathered}
\varepsilon^{0}: D_{0}^{2} u_{0}+u_{0}=0 \\
\varepsilon^{1}: D_{0}^{2} u_{1}+u_{1}=-2 D_{0} D_{1} u_{0}-\mu D_{0} u_{0}+\frac{1}{2} u_{0}^{3} \\
-\frac{\alpha}{4}\left(1-\frac{1}{2} u_{0}^{2}\right) u_{0}^{5}+F \cos (\Omega \tau)
\end{gathered}
$$

The solution of equation (6) can be expressed in the complex form:

$$
u_{0}\left(T_{0}, T_{1}\right)=A\left(T_{1}\right) e^{i T_{0}}+\bar{A}\left(T_{1}\right) e^{-i T_{0}}
$$


where $\bar{A}$ is the complex conjugate of $A$. Substituting the solution (8) into (7)yields to

$$
\begin{aligned}
D_{0} u_{1}+u_{1}= & \left\{-2 i D_{1} A-i \mu A+\frac{3}{2} A^{2} \bar{A}-\frac{\alpha}{4}\left(10 A^{3} \bar{A}^{2}-\frac{35}{2} A^{4} \bar{A}^{3}\right)\right\} e^{i T_{0}} \\
& +\frac{F}{2} e^{i \Omega T_{0}}+N S T+\text { c.c }
\end{aligned}
$$

where NST denotes the terms does not produce secular terms and c.c designates the complex conjugate. Introducing the primary resonance condition given above into (9)and eliminating secular terms, we obtain the following equation:

$$
-2 i A^{\prime}-i \mu A+\frac{3}{2} A^{2} \bar{A}-\frac{\alpha}{4}\left(10 A^{3} \bar{A}^{2}-\frac{35}{2} A^{4} \bar{A}^{3}\right)+\frac{F}{2} e^{i \sigma T_{1}}=0
$$

where the prime (') denotes the derivatives with respect to $T_{1}$.

Writing $A$ in the following polar form:

$$
A=\frac{1}{2} a\left(T_{1}\right) e^{i \theta\left(T_{1}\right)}
$$

where $a\left(T_{1}\right)$ and $\theta\left(T_{1}\right)$ are amplitude and phase response respectively.

Inserting equation (11) into (10) and separating real and imaginary parts, we obtain the modulation equations:

$$
\begin{gathered}
a^{\prime}=\frac{F}{2} \sin \varphi-\frac{\mu}{2} a \\
\varphi^{\prime}=\sigma+\frac{3}{16} a^{2}-\frac{\alpha}{4}\left(\frac{5}{16} a^{4}-\frac{35}{256} a^{6}\right)+\frac{F}{2 a} \cos \varphi
\end{gathered}
$$

where $\varphi=\sigma T_{1}-\theta$,

The steady-state response can be obtained by putting $a^{\prime}=\varphi^{\prime}=0$ in equations (12) and (13). Thus, the steady-state amplitude and phase are governing by the following equations:

$$
\begin{gathered}
\frac{F}{2} \sin \varphi=\frac{\mu}{2} a \\
\frac{F}{2 a} \cos \varphi=-\sigma-\frac{3}{16} a^{2}+\frac{\alpha}{4}\left(\frac{5}{16} a^{4}-\frac{35}{256} a^{6}\right)
\end{gathered}
$$

with $a \neq 0$. For non-trivial solution, eliminating of $\varphi$ from equations (14) and (15), yields to the following equation:

$$
\sigma=-\frac{3}{16} a^{2}+\frac{\alpha}{4}\left(\frac{5}{16} a^{4}-\frac{35}{256} a^{6}\right) \pm \sqrt{\left(\frac{F}{2 a}\right)^{2}-\frac{\mu^{2}}{4}}
$$

Equation (16) represents the frequency-response equation of the primary resonance case. The peak amplitude $a_{p}$ of the system under study at the primary resonance obtained from (16) is given by $a_{p}=\frac{F}{\mu}$ which is inversely proportional to $\mu$. From this equation, we can say that increasing of damping factor $\mu$ can decrease the value of $a_{p}$. Furthermore we notice that the coefficient of quintic- septic nonlinearity does not affect the peak amplitude of the primary resonance response. However the corresponding location of the peak amplitude, depend on $\alpha, \mu$ and $F$ according to the following relationship:

$$
\sigma_{p}=-\frac{F^{2}}{\mu^{2}}\left(\frac{3}{16}-\frac{\alpha}{4}\left(\frac{5 F^{2}}{16 \mu^{2}}-35 F^{3}\right)\right)
$$

Equations (14) and (15) show that is no trivial solution at $a=0$. To determine the stability of the nontrivial solution, let

$$
\begin{aligned}
& a=a_{0}+a_{1} \\
& \varphi=\varphi_{0}+\varphi_{1}
\end{aligned}
$$

where, $a_{1}$ and $\varphi_{1}$ are slight variations. Inserting equations (17) and (18)into equations (12) and (13) and canceling nonlinear terms, we obtain the eigenvalues of the corresponding Jacobian matrix, which are the roots of:

$$
\lambda^{2}+\mu \lambda+P=0
$$

where $P=\frac{\mu^{2}}{4}+\left(\sigma+\frac{3}{16} a^{2}-\frac{5 \alpha}{64} a^{4}\left(1-\frac{7}{16} a^{2}\right)\right)\left(\sigma+\frac{9}{16} a^{2}-\frac{5 \alpha}{64} a^{4}\left(1-\frac{49}{16} a^{2}\right)\right)$

According to the Routh-Wurwitz criteria for stability [30], the steady state solution for the primary resonance case is asymptotically stable if and only if $\mu$ and $\mathrm{P}$ are greater than zero.

The amplitude response curves of the primary resonance case showing the effects of $\alpha, \mu$ and $F$ are plotted in Figures1(a)-(d). In Figure 1(a) we note that when $\alpha$ increases and takes the value 1 , the softening behavior displayed by $\alpha=0$ persists but the corresponding location of the peak amplitude decreases. For high value of $\alpha$ the hardening-softening behavior so-called mixed behavior is obtained with a wide decreasing of location of the peak amplitude. Therefore it appears in the system hysteresis, jump and bifurcation phenomena. For negative value of $\alpha$, the hardening behavior is dominant and softening-hardening behavior is also observed (Figure 1(b)). From these observations we can conclude that the parameter $\alpha$ captures more the nonlinearity of the system understudy and is effectively responsible of mixed behavior. When the excitation amplitude $F$ decreases, the maximum value of the amplitude decreases considerably and hysteresis, jump and bifurcation phenomena disappear in the system (Figure 1(c)). In Figure 1(d) we observe the inverse variations illustrated in Figure1(c). For certain values of the system parameters, the model can exhibit limit cycles whose topology is perturbed by the quintic-septic nonlinearity as illustrated in Figures 2 (a)-(d). In these figures, we observe periodic limit cycles oscillations when $\alpha$ is positive and different shapes of quasi-periodic state signature for negative values of $\alpha$.

\subsection{Other type of Resonant States}

In this subsection, we consider that the periodic external force is wide, that is to say ( $F=\varepsilon^{0} F$ ). In this condition other 
type of oscillations can be displayed by the model, namely sub-harmonic, super-harmonic, sub-super-harmonic and super-sub-harmonic oscillatory states. Applying the multiple time scales method, we obtain the following system:

$$
\begin{gathered}
\varepsilon^{0}: D_{0}^{2} u_{0}+u_{0}=F \cos (\Omega \tau) \\
\varepsilon^{1}: D_{0}^{2} u_{1}+u_{1}=-2 D_{0} D_{1} u_{0}-\mu D_{0} u_{0}+\frac{1}{2} u_{0}^{3} \\
-\frac{\alpha}{4}\left(1-\frac{1}{2} u_{0}^{2}\right) u_{0}^{5}
\end{gathered}
$$

The general solution of equation (20) is:

$$
u_{0}\left(T_{0}, T_{1}\right)=A\left(T_{1}\right) e^{i T_{0}}+\Lambda e^{i \Omega T_{0}}+c . c
$$

where $\Lambda=\frac{F}{2\left(1-\Omega^{2}\right)}$. Substituting (22) into (21) yields to:

$$
\begin{aligned}
D_{0}^{2} u_{1}+u_{1}= & {\left[-2 i D_{1} A-i \mu A+\frac{3}{2} A^{2} \bar{A}+3 \Lambda^{2} A-\frac{\alpha}{4}\left(10 A^{3} \bar{A}^{2}\right.\right.} \\
& +60 \Lambda^{2} A^{2} \bar{A}+30 \Lambda^{4} A-\frac{35}{2} A^{4} \bar{A}^{3}-420 \Lambda^{2} A^{3} \bar{A}^{2} \\
& \left.\left.-315 A^{2} \bar{A} \Lambda^{4}-70 A \Lambda^{6}\right)\right] e^{i T_{0}} \\
& +\left[-i \mu \Omega+3 \Lambda A \bar{A}+\frac{3}{2} \Lambda^{3}-\frac{\alpha}{4}\left(30 \Lambda A^{2} \bar{A}^{2}+60 \Lambda^{3} A \bar{A}\right.\right. \\
& +10 \Lambda^{5}-70 \Lambda A^{3} \bar{A}^{3}-315 \Lambda^{3} A^{2} \bar{A}^{2} \\
& \left.\left.-210 A \bar{A} \Lambda^{5}-\frac{35}{2} \Lambda^{7}\right)\right] e^{i \Omega T_{0}} \\
& +\left[\frac{3}{2} \Lambda \bar{A}^{2}-\frac{\alpha}{4}\left(20 \Lambda A \bar{A}^{3}+30 \Lambda^{3} \bar{A}^{2}-\frac{105}{2} \Lambda A^{2} \bar{A}^{4}\right.\right. \\
& \left.\left.-210 \Lambda^{3} A \bar{A}^{3}-105 \bar{A}^{2} \Lambda^{5}\right)\right] e^{i(\Omega-2) T_{0}} \\
& +\left[\frac{3}{2} \Lambda^{2} \bar{A}-\frac{\alpha}{4}\left(30 \Lambda^{2} A \bar{A}^{2}+20 \Lambda^{4} \bar{A}-\frac{105}{2} \Lambda^{6} \bar{A}\right.\right. \\
& \left.\left.-210 \Lambda^{2} A^{2} \bar{A}^{3}-210 \Lambda^{5} A \bar{A}^{2}\right)\right] e^{i(2 \Omega-1) T_{0}} \\
& +\left[\frac{\Lambda^{3}}{2}-\frac{\alpha}{4}\left(20 \Lambda^{3} A \bar{A}+5 \Lambda^{5}+105 \Lambda^{3} A^{2} \bar{A}^{2}\right.\right. \\
& \left.\left.-105 \Lambda^{5} A \bar{A}-\frac{21}{2} \Lambda^{7}\right)\right] e^{3 i \Omega T_{0}} \\
& -\frac{\alpha}{4}\left[5 \Lambda \bar{A}^{4}-21 \Lambda A \bar{A}^{5}-\frac{105}{2} \Lambda^{3} \bar{A}^{4}\right] e^{i(\Omega-4) T_{0}} \\
& -\frac{\alpha}{4}\left[10 \Lambda^{2} \bar{A}^{3}-105 \Lambda^{2} A \bar{A}^{4}-70 \Lambda^{4} \bar{A}^{3}\right] e^{i(2 \Omega-3) T_{0}} \\
& -\frac{\alpha}{4}\left[10 \Lambda^{3} \bar{A}^{2}-\frac{105}{2} \Lambda^{5} \bar{A}^{2}-70 \Lambda^{3} A \bar{A}^{3}\right] e^{i(3 \Omega-2) T_{0}} \\
& -\frac{\alpha}{4}\left[5 \Lambda^{4} \bar{A}-\frac{35}{2} \Lambda^{4} A \bar{A}^{2}-21 \Lambda^{6} \bar{A}\right] e^{i(4 \Omega-1) T_{0}} \\
& -\frac{\alpha}{4}\left[\Lambda^{5}-\frac{7}{2} \Lambda^{7}-21 \Lambda^{5} A \bar{A}\right] e^{5 i \Omega T_{0}}+\frac{7}{8} \alpha \Lambda \bar{A}^{6} e^{i(\Omega-6) T_{0}} \\
& +\frac{21}{4} \alpha \Lambda^{2} \bar{A}^{5} e^{i(2 \Omega-5) T_{0}}+\frac{21}{8} \alpha \Lambda^{5} \bar{A}^{2} e^{i(5 \Omega-2) T_{0}} \\
& +\frac{7}{8} \bar{A} e^{i(6 \Omega-1) T_{0}}+\frac{35}{8} \alpha \Lambda^{3} \bar{A}^{4} e^{i(3 \Omega-4) T_{0}} \\
& \frac{\alpha}{8} \Lambda^{7} e^{7 i \Omega T_{0}}+N S T+c . c \\
&
\end{aligned}
$$

where "NST" is non secular term and "c. c" denotes the complex conjugate term. Evaluation of (23) shows that the system presents three sub-harmonics, three super-harmonics, three sub-super-harmonics and three super-sub-harmonics resonances states such as:

a) Sub-harmonic

$$
\Omega=3+\varepsilon \sigma ; \Omega=5+\varepsilon \sigma ; \Omega=7+\varepsilon \sigma
$$

b) Super-harmonic

$$
3 \Omega=1+\varepsilon \sigma ; 5 \Omega=1+\varepsilon \sigma \text { and } 7 \Omega=1+\varepsilon \sigma
$$

c) Sub-super-harmonic

$$
2 \Omega=6+\varepsilon \sigma ; 2 \Omega=4+\varepsilon \sigma \text { and } 3 \Omega=5+\varepsilon \sigma
$$

d) Super-sub-harmonic

$$
4 \Omega=2+\varepsilon \sigma ; 5 \Omega=3+\varepsilon \sigma \text { and } 6 \Omega=2+\varepsilon \sigma .
$$

\subsubsection{Sub-harmonic Resonant States}

Considering in this paragraph $\Omega=3+\varepsilon \sigma$, and injecting this condition into (23) and setting secular terms equal to 0 , we obtain:
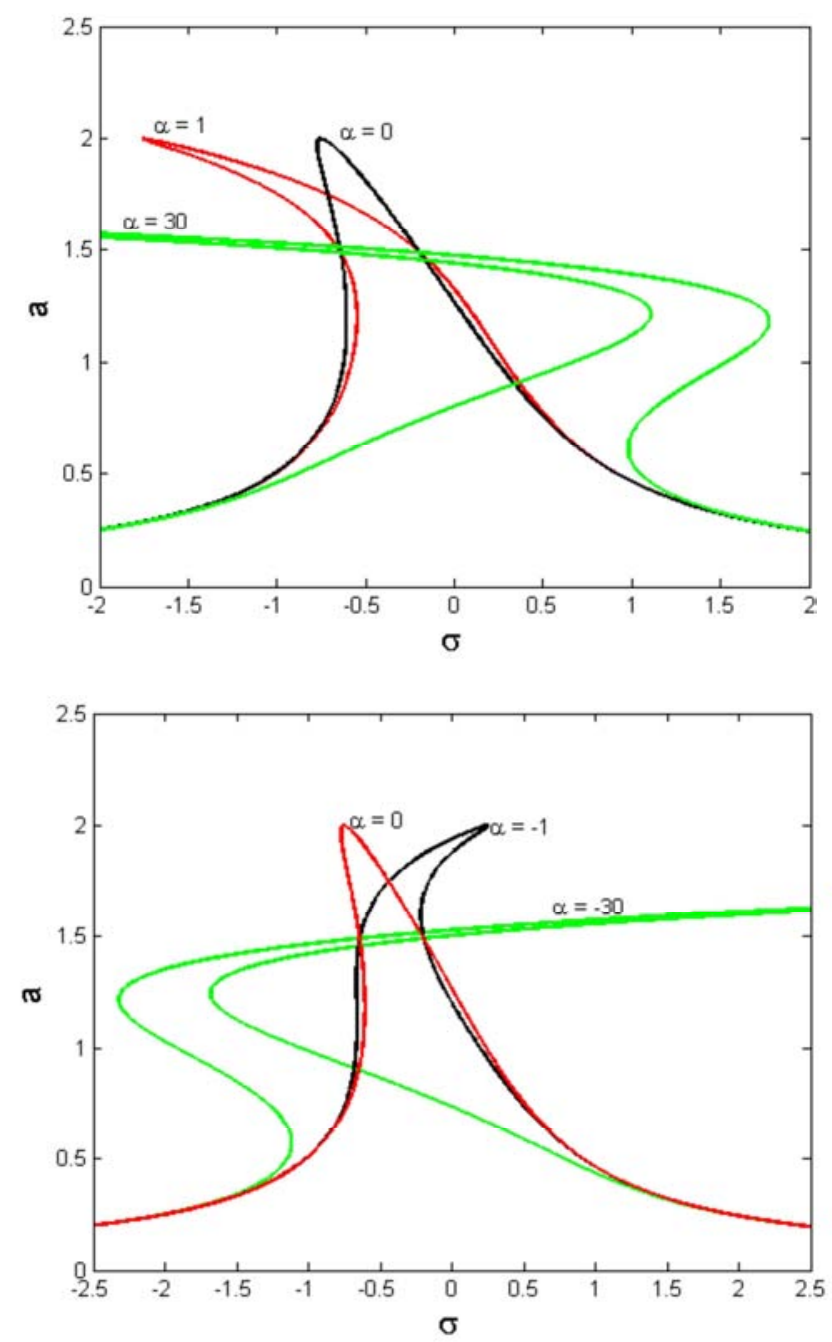

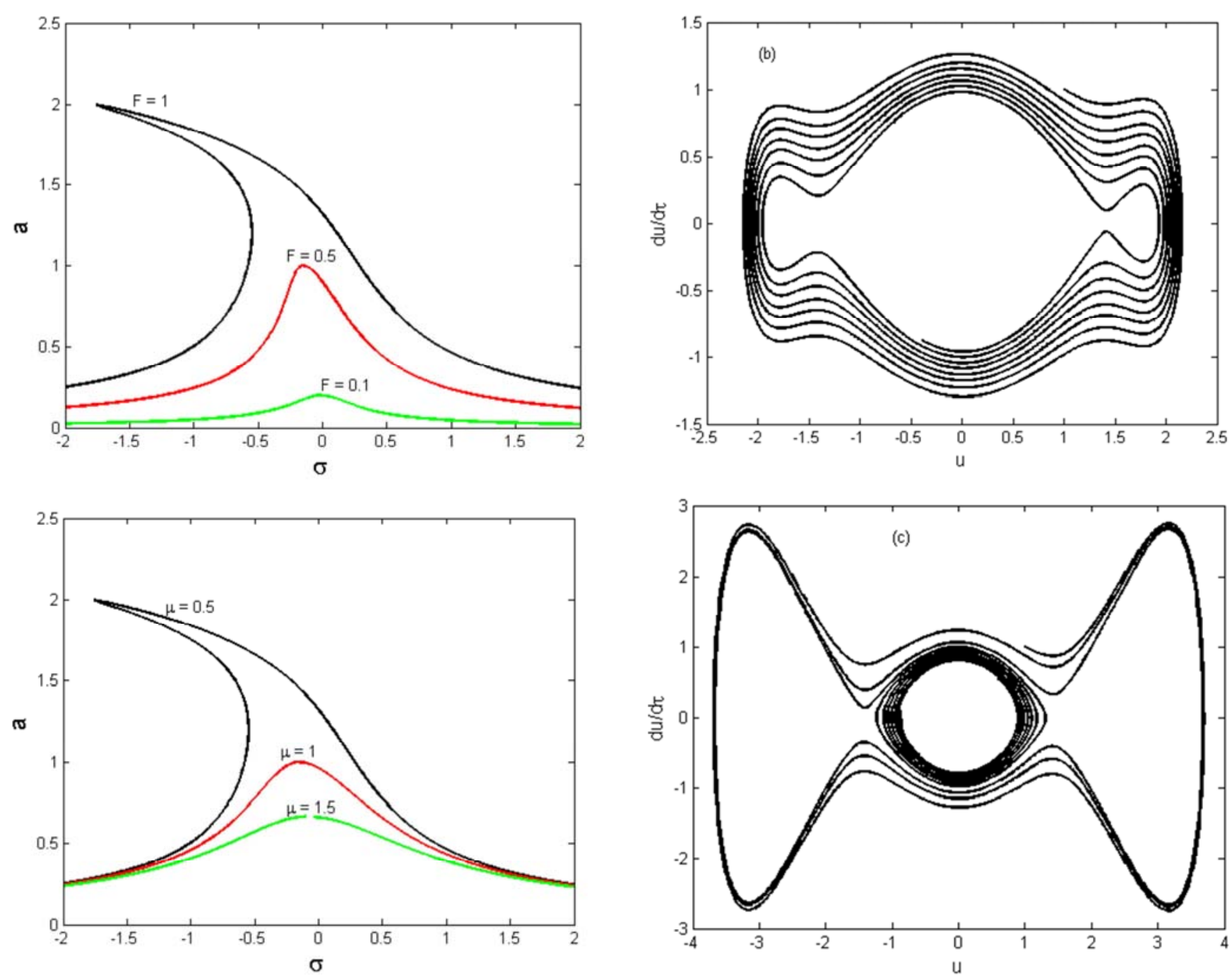

Figure 1. Frequency-response curves for primary resonance showing the effects of: $(a, b) \alpha ;(c) F$ and (d) $\mu$.
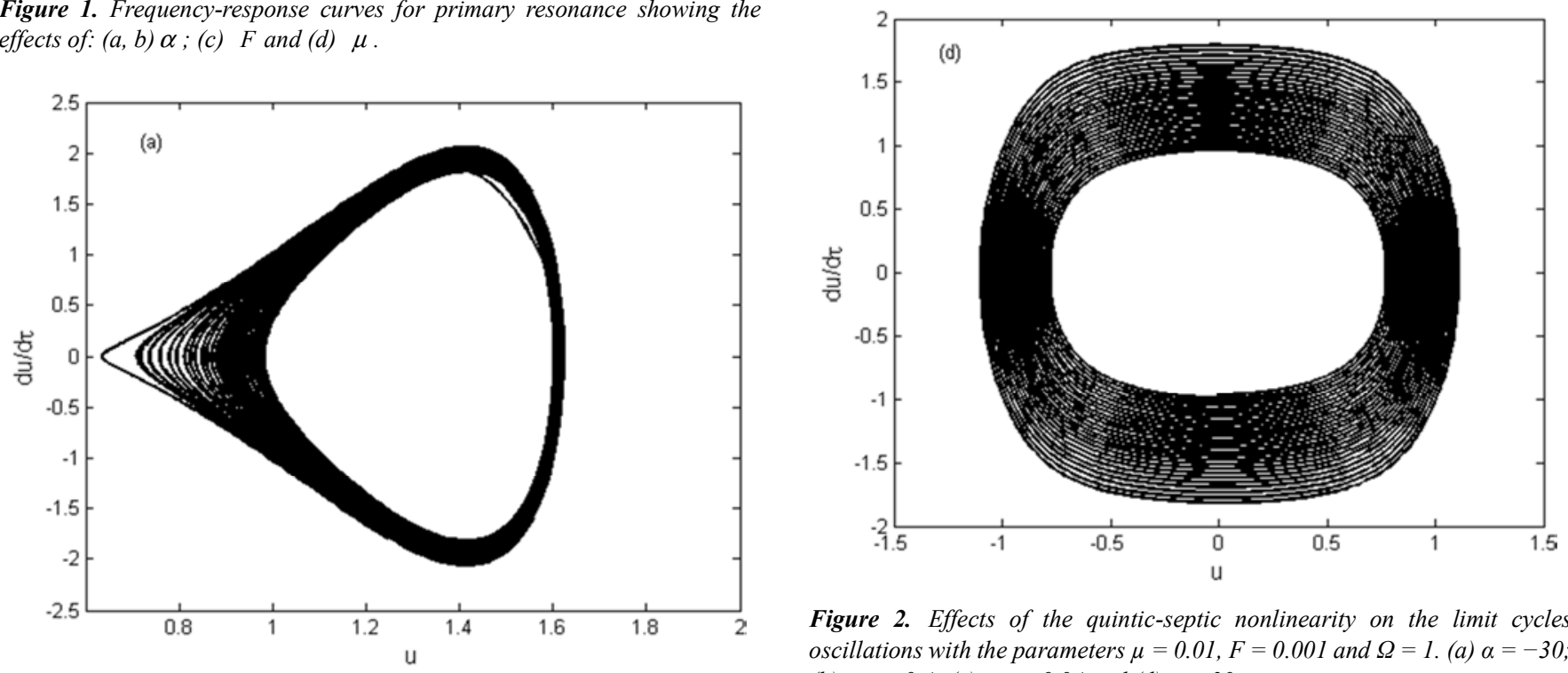

Figure 2. Effects of the quintic-septic nonlinearity on the limit cycles oscillations with the parameters $\mu=0.01, F=0.001$ and $\Omega=1$. (a) $\alpha=-30$; (b) $\alpha=-0.4$; (c) $\alpha=-0.04$ and (d) $\alpha=30$.

$-2 i D_{1} A-i \mu A+\frac{3}{2} A^{2} \bar{A}+3 \Lambda^{2} A-\frac{\alpha}{4}\left(10 A^{3} \bar{A}^{2}+60 \Lambda^{2} A^{2} \bar{A}+30 \Lambda^{4} A-\frac{35}{2} A^{4} \bar{A}^{3}-420 \Lambda^{2} A^{3} \bar{A}^{2}-315 A^{2} \bar{A} \Lambda^{4}-70 A \Lambda^{6}\right)+$ $\left[\frac{3}{2} \Lambda \bar{A}^{2}-\frac{\alpha}{4}\left(20 \Lambda A \bar{A}^{3}+30 \Lambda^{3} \bar{A}^{2}-\frac{105}{2} \Lambda A^{2} \bar{A}^{4}-210 \Lambda^{3} A \bar{A}^{3}-105 \bar{A}^{2} \Lambda^{5}\right)\right] e^{i \sigma T_{1}}=0$ 
Using the polar form into (24) and separating real and imaginary parts, we obtain the following modulation equations:

$$
\begin{aligned}
& a^{\prime}=-\mu \frac{a}{2}+Q \sin \theta \\
& \theta^{\prime}=\sigma-3 Y+\frac{3 Q}{a} \cos \theta
\end{aligned}
$$

where

$$
\begin{aligned}
& Y=-\frac{3 a^{2}}{16}-\frac{3}{2} \Lambda^{2}-\frac{\alpha}{4}\left(\frac{5}{16} a^{4}+\frac{15}{2} \Lambda^{2} a^{2}+15 \Lambda^{4}\right. \\
& \left.-\frac{35}{256} a^{6}-\frac{105}{4} \Lambda^{2} a^{4}-\frac{315}{8} \Lambda^{4} a^{2}-35 \Lambda^{6}\right)
\end{aligned}
$$

and

$$
Q=\frac{3}{8} \Lambda a^{2}-\frac{\alpha}{4}\left(\frac{5}{4} \Lambda a^{4}+\frac{15}{4} \Lambda^{3} a^{2}-\frac{105}{128} \Lambda a^{6}-\frac{105}{8} \Lambda^{3} a^{4}-\frac{105}{4} \Lambda^{5} a^{2}\right)
$$

and

$$
\theta=\sigma T_{1}-3 \beta\left(T_{1}\right)
$$

For steady state solution, $a^{\prime}=\theta^{\prime}=0$, in (25) and (26) we get:

$$
\begin{gathered}
Q \sin \theta=\mu \frac{a}{2} \\
Q \cos \theta=-\frac{\sigma a}{3}+a Y
\end{gathered}
$$

Equations (27) and (28) show that there are two possibilities: (trivial solution) at $a=0$ and (nontrivial solution) at $a \neq 0$. Squaring and adding (27) and (28) we get the frequency -response equation for sub-harmonic resonance of order $\frac{1}{3}$ :

$$
\sigma=3\left(Y \pm \sqrt{\left(\frac{Q}{a}\right)^{2}-\frac{\mu^{2}}{4}}\right)
$$

The stability analysis of the trivial solution is equivalent to the analysis of the linear solution of equation (24) by neglecting the non-linear terms. Thus, we obtain:

$$
-2 i A^{\prime}-i \mu A+\Lambda^{2} A\left(3+30 \Lambda^{2}-70 \Lambda^{4}\right)=0
$$

To solve (30), we inject $A=e^{\frac{1}{2} i \sigma T_{1}}\left(B\left(T_{1}\right)+i b\left(T_{1}\right)\right)$

where $B$ and $b$ are real into equation (30). Separating real and imaginary parts, we get:

$$
B^{\prime}=-\frac{\mu}{2} B+\frac{1}{2}\left[\sigma+3 \Lambda^{2}-\frac{5}{2} \alpha \Lambda^{4}\left(3-7 \Lambda^{2}\right)\right] b
$$

$$
b^{\prime}=-\frac{1}{2}\left[\sigma+3 \Lambda^{2}-\frac{5}{2} \alpha \Lambda^{4}\left(3-7 \Lambda^{2}\right)\right] B-\frac{\mu}{2} b
$$

The eigenvalues of the Jacobian matrix satisfy the equation:

$$
\lambda^{2}+\mu \lambda+P_{2}=0
$$

where

$$
P_{2}=\frac{\mu^{2}}{4}+\frac{1}{4}\left[\sigma+3 \Lambda^{2}-\frac{5}{2} \alpha \Lambda^{4}\left(3-7 \Lambda^{2}\right)\right]^{2} .
$$

According to the Routh-Wurwitz criteria for stability, the trivial steady-state solution for the sub-harmonic resonance of $\operatorname{order} \frac{1}{3}$ is asymptotically stable since $\mu>0$ and $P_{2}>0$.

The stability of the nontrivial steady-state sub-harmonic resonance of order $\frac{1}{3}$ is determined by the eigenvalues of the corresponding Jacobian matrix, which are the roots of

$$
\lambda^{2}+P_{3} \lambda+P_{4}=0
$$

where

$$
P_{3}=\mu\left(2-\frac{Q^{\prime}\left(a_{0}\right)}{2 Q\left(a_{0}\right)} a_{0}\right)
$$

and

$$
P_{4}=\left(1-\frac{Q^{\prime}\left(a_{0}\right)}{Q\left(a_{0}\right)} a_{0}\right)\left(\frac{3}{4} \mu^{2}+\frac{1}{3}\left(3 Y\left(a_{0}\right)-\sigma\right)^{2}\right)+a_{0}\left(3 Y\left(a_{0}\right)-\sigma\right) Y^{\prime}\left(a_{0}\right)
$$

According to the Routh-Wurwitz criteria for stability, the nontrivial steady-state solution for the sub-harmonic resonance of order $1 / 3$ is asymptotically stable if and only if $P_{3}>0$ and $P_{4}>0$. The frequency-response curves for sub-harmonic resonance of order1/3illustrating the effects of the parameters $\alpha, F$ and are represented in Figures 3(a)-(d). In Figure 3(a) we observe that when $\alpha$ increases the softening behavior observed in the case $\alpha=0$ change into hardening behavior, that is to say the response amplitude of the $\frac{1}{3}$ sub-harmonic resonance, which consist of two branches move to the right. Then it appears in the system jump and bifurcation phenomena. It is important to point out that this increasing of $\alpha$ affect the maximum value of the response amplitude and the location of minimum value of the response amplitude. For negative value of $\alpha$ the softening behavior is dominant but the location of minimum value of the response amplitude increases while the maximum amplitude value decreases (Figure 3(b)). From Figure 3(c) we note that jump and bifurcation phenomena exist since the hardening behavior is obtained. Moreover the resonance bandwidth and the location of minimum value of the response amplitude increase with increasing of $F$. However the maximum 
response amplitude decreases when the excitation amplitude $F$ increases. When $\mu$ increases, the minimum value of the response amplitude highly increases but this variation of $\mu$ has no effect on the maximum value of the $\frac{1}{3}$ sub-harmonic resonance response (Figure 3(d)).
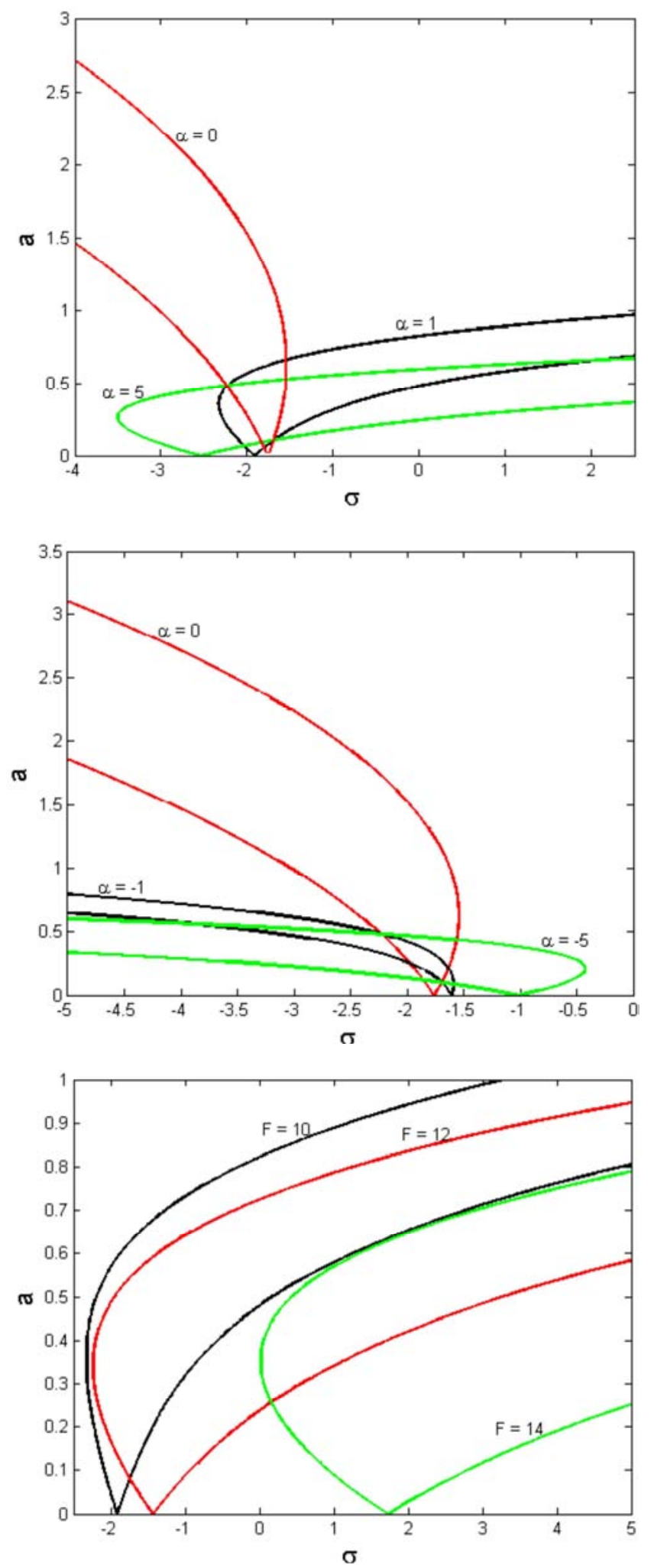

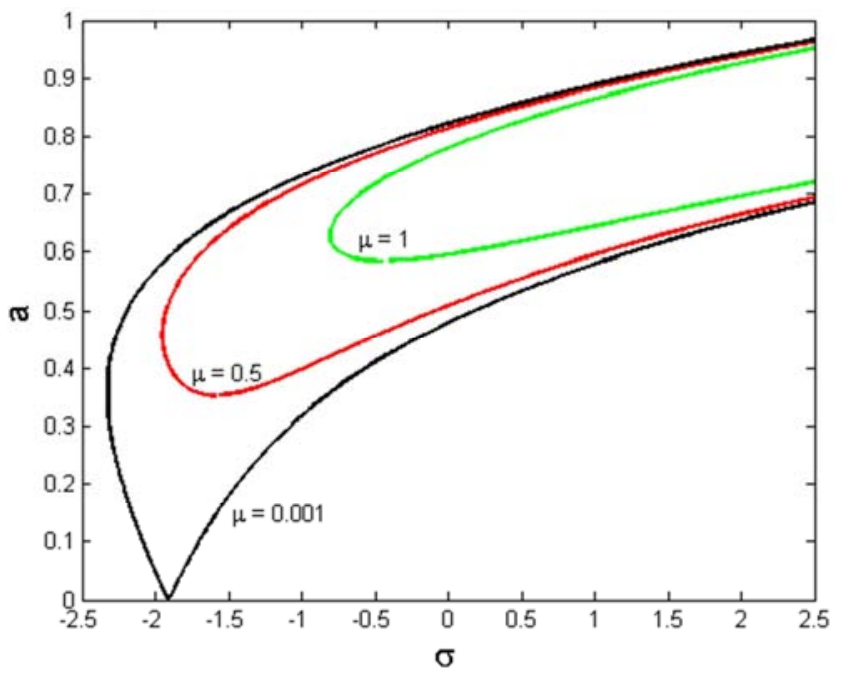

Figure 3. Frequency-response curves for $1 / 3$ sub-harmonic resonance illustrating the effects of: $(a, b) \alpha$; (c) $F$ and (d) $\mu$.

Considering the case $\Omega=5+\varepsilon \sigma$, and inserting this relation into (23) and eliminating secular terms, we get

$$
\begin{array}{r}
-2 i D_{1} A-i \mu A+\frac{3}{2} A^{2} \bar{A}+3 \Lambda^{2} A-\frac{\alpha}{4}\left(10 A^{3} \bar{A}^{2}\right. \\
+60 \Lambda^{2} A^{2} \bar{A}+30 \Lambda^{4} A-\frac{35}{2} A^{4} \bar{A}^{3}-420 \Lambda^{2} A^{3} \bar{A}^{2} \\
\left.\quad-315 A^{2} \bar{A} \Lambda^{4}-70 A \Lambda^{6}\right) \\
-\frac{\alpha}{4}\left[5 \Lambda \bar{A}^{4}-21 \Lambda A \bar{A}^{5}-\frac{105}{2} \Lambda^{3} \bar{A}^{4}\right] e^{i \sigma T_{1}}=0
\end{array}
$$

Using the polar form $A=\frac{1}{2} a\left(T_{1}\right) e^{i \beta\left(T_{1}\right)}$ into equation (35) and separating real and imaginary parts, we obtain the following modulation equations:

$$
\begin{gathered}
a^{\prime}=-\mu \frac{a}{2}+R \sin \theta \\
\theta^{\prime}=\sigma-5 Y+\frac{5 R}{a} \cos \theta
\end{gathered}
$$

where

$$
R=-\frac{\alpha}{4}\left[\frac{5 \Lambda}{16} a^{4}\left(1-\frac{21 \Lambda^{2}}{2}\right)-\frac{21 \Lambda}{64} a^{6}\right] \text { and } \theta=\sigma T_{1}-5 \beta\left(T_{1}\right)
$$

Introducing the steady-state condition $a^{\prime}=\theta^{\prime}=0$, into equations (36) and (37), we obtain:

$$
\begin{gathered}
R \sin \theta=\mu \frac{a}{2} \\
R \cos \theta=-\frac{\sigma a}{5}+a Y
\end{gathered}
$$


Equations (38) and (39) show that there are two possibilities: (trivial solution) at $a=0$ and (nontrivial solution) at $a \neq 0$. The stability of the trivial steady-state sub-harmonic resonance of order $\frac{1}{5}$ is the same that those examined in the case of the sub-harmonic resonance of order $\frac{1}{3}$.

Squaring and adding (38) and (39) we get after some mathematical manipulations the following frequency-response equation of the sub-harmonic resonance of $\operatorname{order} \frac{1}{5}$ :

$$
\sigma=5\left(Y \pm \sqrt{\left(\frac{R}{a}\right)^{2}-\frac{\mu^{2}}{4}}\right)
$$

The stability of the nontrivial steady-state sub-harmonic resonance of order $\frac{1}{5}$ is examined by the eigenvalues of the corresponding Jacobian matrix, which are the roots of

$$
\lambda^{2}+P_{5} \lambda+P_{6}=0
$$

where

$$
P_{5}=\mu\left(3-\frac{R^{\prime}\left(a_{0}\right)}{2 R\left(a_{0}\right)} a_{0}\right)
$$

and

$$
P_{6}=\left(1-\frac{R^{\prime}\left(a_{0}\right)}{R\left(a_{0}\right)} a_{0}\right)\left(\frac{3}{4} \mu^{2}+\frac{1}{5}\left(5 Y\left(a_{0}\right)-\sigma\right)^{2}\right)+a_{0}\left(5 Y\left(a_{0}\right)-\sigma\right) Y^{\prime}\left(a_{0}\right)
$$

If the two inequalities $P_{5}>0$ and $P_{6}>0$ are satisfied then the steady-state solution will be asymptotically stable.

Figures 4(a)-(c) present the effects of $\alpha$ and $F$ on the sub-harmonic resonance solution of order $\frac{1}{5}$. In Figure 4(a) we note that the frequency-response curves of this case of oscillation is obtained for high positive values of detuning parameter. In this figure we observe that when $\alpha$ increases positively the response amplitude curves move to the right with a large increasing of the resonance frequency. When $\alpha$ takes negative value the obtained variations are contrary to those shown in Figure 3(b). From Figure 4(c) we note the same variations as illustrated in Figure 3(a) but with a high value of excitation amplitude.

\subsubsection{Super-harmonic Resonant States}

Using the super-harmonic resonance relation $3 \Omega=1+\varepsilon \sigma$ into equation (23), the condition for eliminating secular terms in the problem is given by:

$$
\begin{aligned}
& -2 i D_{1} A-i \mu A+\frac{3}{2} A^{2} \bar{A}+3 \Lambda^{2} A-\frac{\alpha}{4}\left(10 A^{3} \bar{A}^{2}+60 \Lambda^{2} A^{2} \bar{A}+30 \Lambda^{4} A-\frac{35}{2} A^{4} \bar{A}^{3}-420 \Lambda^{2} A^{3} \bar{A}^{2}-315 \Lambda^{4} A^{2} \bar{A}-70 A \Lambda^{6}\right) \\
& +\left[\frac{\Lambda^{3}}{2}-\frac{\alpha}{4}\left(20 \Lambda^{3} A \bar{A}+5 \Lambda^{5}+105 \Lambda^{3} A^{2} \bar{A}^{2}-105 \Lambda^{5} A \bar{A}-\frac{21}{2} \Lambda^{7}\right)\right] e^{i \sigma T_{1}}=0
\end{aligned}
$$

Using the polar form $A=\frac{1}{2} a\left(T_{1}\right) e^{i \beta\left(T_{1}\right)}$ into equation and separating real and imaginary parts, we obtain the following modulation equations:

$$
\begin{gathered}
a^{\prime}=-\mu \frac{a}{2}+M \sin \theta \\
\theta^{\prime}=\sigma-Y+\frac{M}{a} \cos \theta
\end{gathered}
$$

Where

$M=\frac{\Lambda^{3}}{2}-\frac{\alpha}{4}\left(5 \Lambda^{3} a^{2}+5 \Lambda^{5}+\frac{105}{16} \Lambda^{3} a^{4}-\frac{105}{4} \Lambda^{5} a^{2}-\frac{21}{2} \Lambda^{7}\right)$ and $\theta=\sigma T_{1}-\beta\left(T_{1}\right)$.

Using the steady-state solution, $a^{\prime}=\theta^{\prime}=0$ in equations (43) and (44), we get:

$$
\begin{array}{r}
M \sin \theta=\mu \frac{a}{2} \\
M \cos \theta=-\sigma a+a Y
\end{array}
$$

After a few algebraic operations we obtain the frequency-response equation for super-harmonic resonance of order 3 .

$$
\sigma=Y \pm \sqrt{\left(\frac{M}{a}\right)^{2}-\frac{\mu^{2}}{4}}
$$

From equations (45) and (46) we notice that there only exists non-trivial solution at $a \neq 0$.

The stability of the steady-state super-harmonic resonance of order 3 is determined by the eigenvalues of the corresponding Jacobian matrix, which are the roots of

$$
\lambda^{2}+P_{7} \lambda+P_{8}=0
$$

where

$$
P_{7}=\mu\left(1-\frac{M^{\prime}\left(a_{0}\right)}{2 M\left(a_{0}\right)} a_{0}\right)
$$

and

$$
P_{8}=\left(1-\frac{M^{\prime}\left(a_{0}\right)}{M\left(a_{0}\right)} a_{0}\right)\left(\frac{1}{4} \mu^{2}+\left(Y\left(a_{0}\right)-\sigma\right)^{2}\right)+a_{0}\left(Y\left(a_{0}\right)-\sigma\right) Y^{\prime}\left(a_{0}\right)
$$



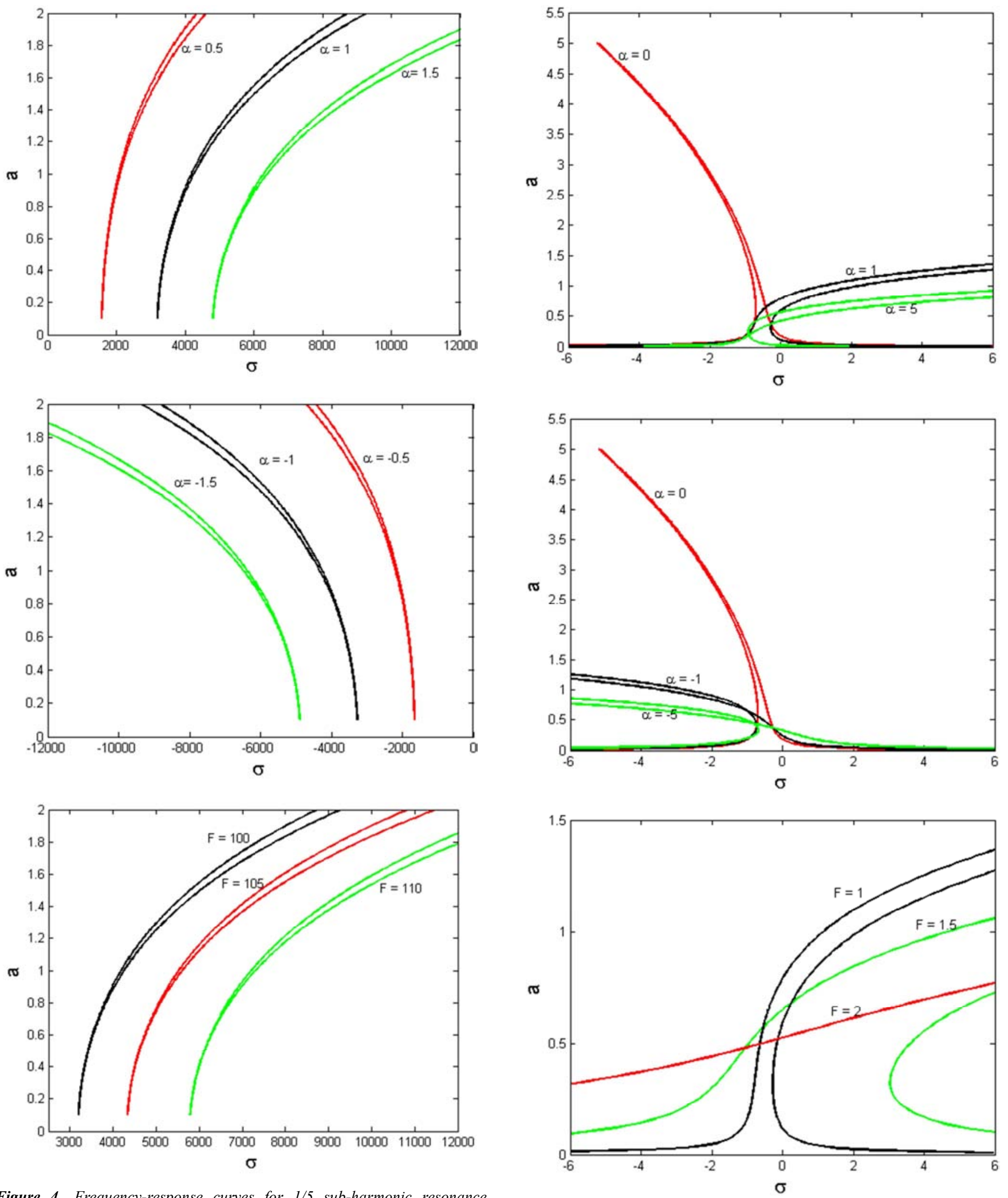

Figure 4. Frequency-response curves for $1 / 5$ sub-harmonic resonance exhibiting the effects of: $(a, b) \alpha$ and (c) $F$.

According to the Routh-Wurwitz criteria for stability, the steady-state super-harmonic resonance of order 3 is asymptotically stable if and only if $P_{7}>0$ and $P_{8}>0$.

Figure 5. Frequency-response curves for super-harmonic resonance of order 3 showing the effects of: $(a, b) \alpha$ and (c) $F$.

Figures 5(a)-(c) show the effects of $\alpha$ and $F$ on the super-harmonic resonance response of order 3. In Figure 5(a), we notice that when $\alpha$ takes the value 1 the response amplitude consists of two curves move to the right. Therefore 
hardening behavior is obtained and jump and bifurcation phenomena exist. However the hysteresis phenomenon disappears in the system. Furthermore increasing of $\alpha$ reduced considerably the maximum value of the response amplitude and when $\alpha=5$, the resonance curves touch at one location. At this location, the corresponding amplitude is critical. For negative value of $\alpha$ softening behavior becomes dominant with decreasing of the response amplitude. The resonance curves touch at different locations and become open like those of the undamped oscillator (Figure 5(b)). From Figure 5(c) we note that increasing of $F$ decreases the maximum value of the response amplitude but the resonance bandwidth increases. Moreover we observe that when $F=2$ the lower branch of the super-harmonic resonance of order 3 disappears on the considered interval for detuning parameter $\sigma$.

Another case of oscillation where $5 \Omega=1+\varepsilon \sigma$ is treated in this part. Injecting this condition into (23) and eliminating the resulting secular we get:

$$
-2 i D_{1} A-i \mu A+\frac{3}{2} A^{2} \bar{A}+3 \Lambda^{2} A-\frac{\alpha}{4}\left(10 A^{3} \bar{A}^{2}+60 \Lambda^{2} A^{2} \bar{A}+30 \Lambda^{4} A-\frac{35}{2} A^{4} \bar{A}^{3}-420 \Lambda^{2} A^{3} \bar{A}^{2}-315 \Lambda^{4} A^{2} \bar{A}-70 A \Lambda^{6}\right)-\frac{\alpha}{4}\left(\Lambda^{5}-\frac{7 \Lambda^{7}}{2}-21 \Lambda^{5} A \bar{A}\right) e^{i \sigma T_{1}}=0
$$

Inserting the polar form of the $A$ into (49) and separating real and imaginary parts, we obtain the following modulation equations:

$$
\begin{gathered}
a^{\prime}=-\mu \frac{a}{2}+S \sin \theta \\
\theta^{\prime}=\sigma-Y+\frac{M}{a} \cos \theta
\end{gathered}
$$

where

$$
S=-\frac{\alpha}{4}\left(\Lambda^{5}-\frac{21}{4} \Lambda^{5} a^{2}-\frac{7}{2} \Lambda^{7}\right)
$$

and $\theta=\sigma T_{1}-\beta\left(T_{1}\right)$

Introducing the steady-state solution, $a^{\prime}=\theta^{\prime}=0$, into equations (50) and (51), we get:

$$
\begin{gathered}
S \sin \theta=\mu \frac{a}{2} \\
S \cos \theta=-\sigma a+a Y
\end{gathered}
$$

Eliminating of $\theta$ from this equation systems yields to after a few algebraic manipulations the following frequency-response equation for super-harmonic resonance of order 5:

$$
\sigma=Y \pm \sqrt{\left(\frac{S}{a}\right)^{2}-\frac{\mu^{2}}{4}}
$$

The stability of the steady-state 5:1super-harmonic resonance response is determined by the eigenvalues of the corresponding Jacobian matrix, which are the roots of

$$
\lambda^{2}+P_{9} \lambda+P_{10}=0
$$

where

$$
P_{9}=\mu\left(1-\frac{S^{\prime}\left(a_{0}\right)}{2 S\left(a_{0}\right)} a_{0}\right)
$$

And $P_{10}=\left(1-\frac{S^{\prime}\left(a_{0}\right)}{S\left(a_{0}\right)} a_{0}\right)\left(\frac{1}{4} \mu^{2}+\left(Y\left(a_{0}\right)-\sigma\right)^{2}\right)+a_{0}\left(Y\left(a_{0}\right)-\sigma\right) Y^{\prime}\left(a_{0}\right)$

According to the Routh-Wurwitz criteria for stability, the non-trivial steady-state solution of the super-harmonic resonance of order 5 is asymptotically stable if and only if $P_{9}>0$ and $P_{10}>0$.

The frequency-response curves for super-harmonic resonance of order5 exhibiting the effects of $\alpha$ and $F$ is presented in Figures 6(a)-(c). In this case of oscillation, the response amplitude is obtained for large value of detuning parameter. From Figures 6(a) and (c), the response amplitude consists of two curves which move to the right and have hardening behavior. Therefore jump and bifurcation phenomena exist in the system. For negative value of $\alpha$ resonance curves move to the left pointing out that the system displays the softening behavior (Figure $6(\mathrm{~b})$ ).

\subsubsection{Sub-super-and Super-sub-harmonic Resonant States}

In this paragraph we consider the case of sub-super-harmonic resonance $2 \Omega \cong 4$ and therefore set $2 \Omega=4+\varepsilon \sigma$. Eliminating secular terms from (23), we obtain the following equation:

$$
\begin{aligned}
& -2 i D_{1} A-i \mu A+\frac{3}{2} A^{2} \bar{A}+3 \Lambda^{2} A-\frac{\alpha}{4}\left(10 A^{3} \bar{A}^{2}+60 \Lambda^{2} A^{2} \bar{A}+30 \Lambda^{4} A\right. \\
& \left.-\frac{35}{2} A^{4} \bar{A}^{3}-420 \Lambda^{2} A^{3} \bar{A}^{2}-315 \Lambda^{4} A^{2} \bar{A}-70 A \Lambda^{6}\right) \\
& -\frac{\alpha}{4}\left(10 \Lambda^{2} \bar{A}^{3}-105 \Lambda^{2} A \bar{A}^{4}-70 \Lambda^{4} \bar{A}^{3}\right) e^{i \sigma T_{1}}=0
\end{aligned}
$$

Injecting the polar form of the $A$ into equation (56) and separating real and imaginary parts, we obtain the following modulation equations:

$$
\begin{aligned}
& a^{\prime}=-\mu \frac{a}{2}+L \sin \theta \\
& \theta^{\prime}=\sigma-4 Y+\frac{4 L}{a} \cos \theta
\end{aligned}
$$

where

$$
L=-\frac{5 \alpha \Lambda^{2} a^{3}}{16}\left(1-\frac{21}{8} a^{2}-7 \Lambda^{2}\right) \text { and } \theta=\sigma T_{1}-4 \beta\left(T_{1}\right)
$$

For steady state solution, $a^{\prime}=\theta^{\prime}=0$, in equations (57) and (58), we obtain: 


$$
\begin{gathered}
L \sin \theta=\mu \frac{a}{2} \\
L \cos \theta=-\sigma \frac{a}{4}+a Y
\end{gathered}
$$

Equations (59) and (60) show that there are two possibilities: (trivial solution) at $a=0$ and (nontrivial solution) at $a \neq 0$. Squaring and adding (59) and (60) we get the following frequency-response equation after a few mathematical manipulations:

$$
\sigma=4\left(Y \pm \sqrt{\left(\frac{L}{a}\right)^{2}-\frac{\mu^{2}}{4}}\right)
$$

The stability of the trivial sub-super-harmonic resonance of order $\frac{1}{2}$ is the same that those obtained in the case of the sub-harmonic resonance of $\operatorname{order} \frac{1}{3}$. However the stability of the nontrivial steady-state response is analyzed by the eigenvalues of the corresponding Jacobian matrix, which are roots of

$$
\lambda^{2}+P_{11} \lambda+P_{12}=0
$$

where

$$
\begin{gathered}
P_{11}=\mu\left(\frac{5}{2}-\frac{L^{\prime}\left(a_{0}\right)}{2 L\left(a_{0}\right)} a_{0}\right) \\
P_{12}=\frac{1}{4}\left(1-\frac{L^{\prime}\left(a_{0}\right)}{L\left(a_{0}\right)} a_{0}\right)\left(\mu^{2}+\left(Y\left(a_{0}\right)-\sigma\right)^{2}\right)+a_{0}\left(Y\left(a_{0}\right)-\sigma\right) Y^{\prime}\left(a_{0}\right)
\end{gathered}
$$

According to the Routh-Wurwitz criteria for stability, the steady-state solution of the sub-super-harmonic resonance of order $\frac{1}{2}$ is asymptotically stable if and only if $P_{11}>0$ and $P_{12}>0$. The frequency-response curves for sub-super-harmonic resonance of order $\frac{1}{2}$ displaying the effects of $\alpha$ and $F$ are represented in Figures 7(a)-(c). In Figure 7 (a) we observe that the response amplitude is obtained for high positive values of detuning parameter. This response composed of two branches move to the right. Then hardening behavior and bifurcation phenomenon exist in the system. Furthermore when $\alpha$ increases the resonance bandwidth and the resonance frequency increase. When $\alpha$ takes negative value the softening behavior is dominant in the system (Figure 7(b)). In Figure 7(c) when $F$ increases, we notice the same variations illustrated in Figure 7(a).
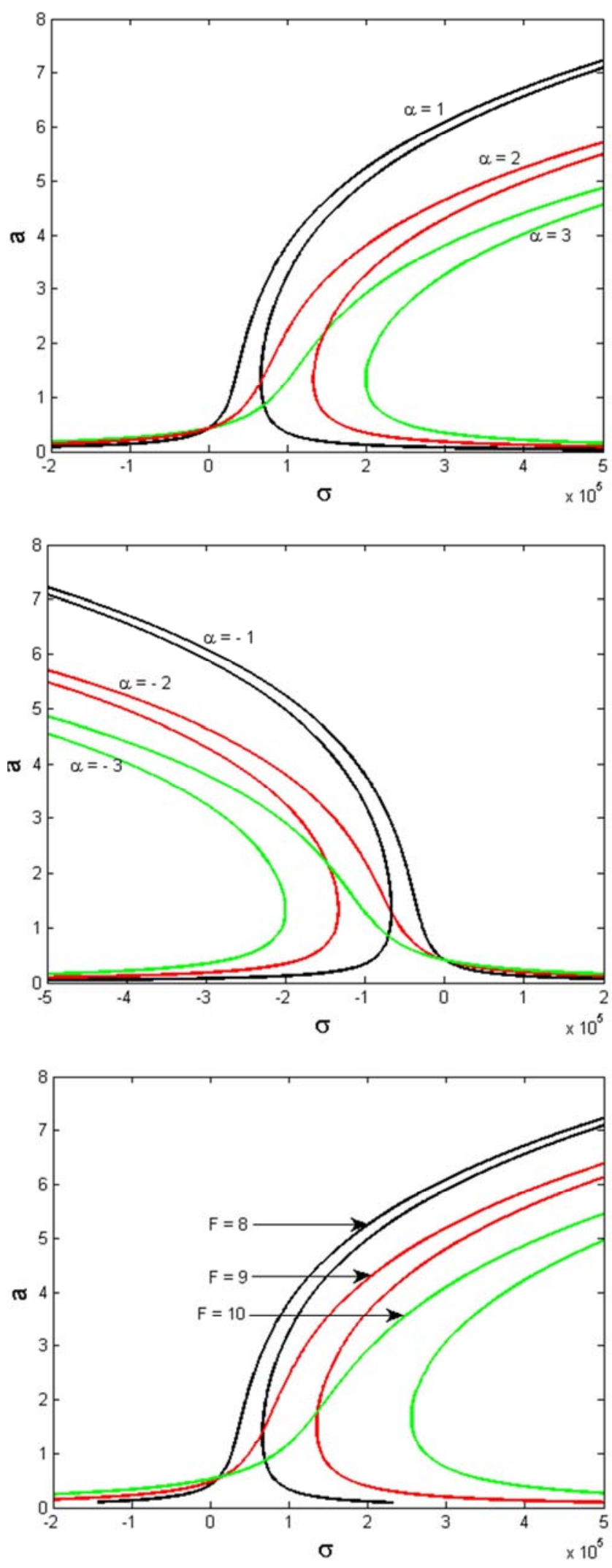

Figure 6. Frequency-response curves for super-harmonic resonance of order 5 showing the effects of: $(a, b) \alpha$ and (c) $F$. 

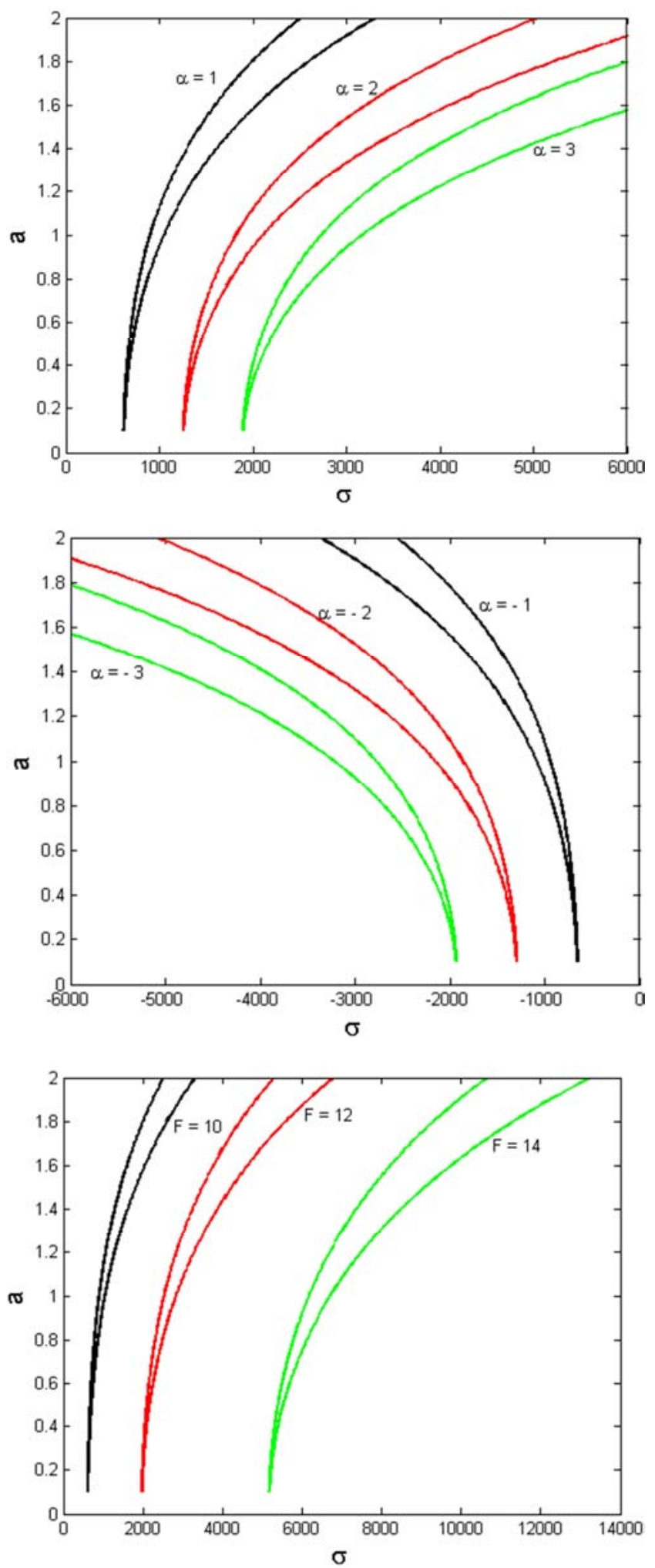

Figure 7. Frequency-response curves for 1/2 sub-super-harmonic resonance exhibiting the effects of: $(a, b) \alpha$ and (c) $F$.

At present we consider the case of super-sub-harmonic resonance $4 \Omega \cong 2$ and therefore set $4 \Omega=2+\varepsilon \sigma$. Eliminating secular terms from (23), we obtain the following equation:

$$
\begin{aligned}
& -2 i D_{1} A-i \mu A+\frac{3}{2} A^{2} \bar{A}+3 \Lambda^{2} A-\frac{\alpha}{4}\left(10 A^{3} \bar{A}^{2}+60 \Lambda^{2} A^{2} \bar{A}+30 \Lambda^{4} A\right. \\
& \left.-\frac{35}{2} A^{4} \bar{A}^{3}-420 \Lambda^{2} A^{3} \bar{A}^{2}-315 \Lambda^{4} A^{2} \bar{A}-70 A \Lambda^{6}\right) \\
& -\frac{\alpha}{4}\left(5 \Lambda^{4} \bar{A}-\frac{105}{2} \Lambda^{4} A \bar{A}^{2}-21 \Lambda^{6} \bar{A}\right) e^{i \sigma T_{1}}=0
\end{aligned}
$$

Inserting the polar form of the A into (63) and separating real and imaginary parts, we obtain the following modulation equations:

$$
\begin{gathered}
a^{\prime}=-\mu \frac{a}{2}+K \sin \theta \\
\theta^{\prime}=\sigma-2 Y+\frac{2 K}{a} \cos \theta
\end{gathered}
$$

where,

$$
K=-\frac{\alpha}{4}\left[\frac{5}{2} \Lambda^{4} a-\frac{105}{16} \Lambda^{4} a^{3}-\frac{21}{2} \Lambda^{6} a\right] \text { and } \theta=\sigma T_{1}-2 \beta\left(T_{1}\right)
$$

For steady-state solution, $a^{\prime}=\theta^{\prime}=0$, in (64) and (65) we obtain:

$$
\begin{gathered}
K \sin \theta=\mu \frac{a}{2} \\
K \cos \theta=-\sigma \frac{a}{2}+a Y
\end{gathered}
$$

Equations (66) and (67) show that there are two possibilities: (trivial solution) at $a=0$ and (nontrivial solution) at $a \neq 0$. Squaring and adding (66) and (67), we get after a few mathematical operations the following frequency-response equation:

$$
\sigma=2\left(Y \pm \sqrt{\left(\frac{K}{a}\right)^{2}-\frac{\mu^{2}}{4}}\right)
$$

The stability analysis of the trivial solution is equivalent to the analysis of the linear solution of (63) by neglecting the non-linear terms. Thus we get:

$$
-2 i A^{\prime}-i \mu A+3 \Lambda^{2} A-\frac{\alpha}{4}\left[30 \Lambda^{4} A-70 \Lambda^{6} A+\left(5 \Lambda^{4} \bar{A}-21 \Lambda^{6} \bar{A}\right) e^{i \sigma T_{1}}\right]=0
$$

To solve (69) one lets $A=e^{\frac{i}{2} \sigma T_{1}}\left(B\left(T_{1}\right)+i b\left(T_{1}\right)\right)$ where $B$ and $b$ are real and imaginary parts, and we get after inserting $A$ into (69), the following linear equations system:

$$
\begin{gathered}
B^{\prime}=-\frac{\mu}{2} B+\frac{1}{2}\left[\sigma+3 \Lambda^{2}-\frac{\alpha}{4} \Lambda^{4}\left(35-91 \Lambda^{2}\right)\right] b \\
b^{\prime}=-\frac{1}{2}\left[\sigma+3 \Lambda^{2}-\frac{\alpha}{4} \Lambda^{4}\left(35-91 \Lambda^{2}\right)\right] B \quad-\frac{\mu}{2} b
\end{gathered}
$$

The characteristic equation of the corresponding Jacobian 
matrix is given by

$$
\lambda^{2}+\mu \lambda+P_{13}=0
$$

where

$$
P_{13}=\frac{\mu^{2}}{4}+\frac{1}{4}\left[\sigma+3 \Lambda^{2}-\frac{\alpha}{4} \Lambda^{4}\left(35-91 \Lambda^{2}\right)\right]^{2}
$$

According to the Routh-Wurwitz criteria for stability, the trivial solution of the super-sub-harmonic resonance of order 2 is asymptotically stable since $P_{13}>0$ and $\mu$ is here positive. The stability of the nontrivial steady-state super-sub-harmonic resonance of order 2 given by (68) is determined by the eigenvalues of the characteristic equation, which are the roots of:

$$
\lambda^{2}+P_{14} \lambda+P_{15}=0
$$

where $P_{14}=\frac{\mu}{2}\left(5-\frac{K^{\prime}\left(a_{0}\right)}{K\left(a_{0}\right)} a_{0}\right)$

and

$$
P_{15}=\frac{1}{2}\left(1-\frac{K^{\prime}\left(a_{0}\right)}{K\left(a_{0}\right)} a_{0}\right)\left(\frac{1}{2} \mu^{2}+\left(Y\left(a_{0}\right)-\sigma\right)^{2}\right)+a_{0}\left(Y\left(a_{0}\right)-\sigma\right) Y^{\prime}\left(a_{0}\right)
$$

The nontrivial steady-state solution for the super-sub-harmonic resonance of order 2 is asymptotically stable if and only if $P_{14}>0$ and $P_{15}>0$. Figures 8(a)-(c) represent the effects of $\alpha$ and $F$ on the super-sub-harmonic resonance of order 2. In this oscillation case, the response amplitude is obtained for high positive values. In Figure 8(a) and (c), the response amplitude consists of two branches which bend to the right and have hardening nonlinearity. Therefore it appears in the system jump and bifurcation phenomena. However the hysteresis phenomenon does not exist. In these figures, when $\alpha$ and $\mathrm{F}$ increase, the resonance bandwidth increases. For negative value of $\alpha$, the response amplitude displays a softening behavior (see Figure 8(b)).

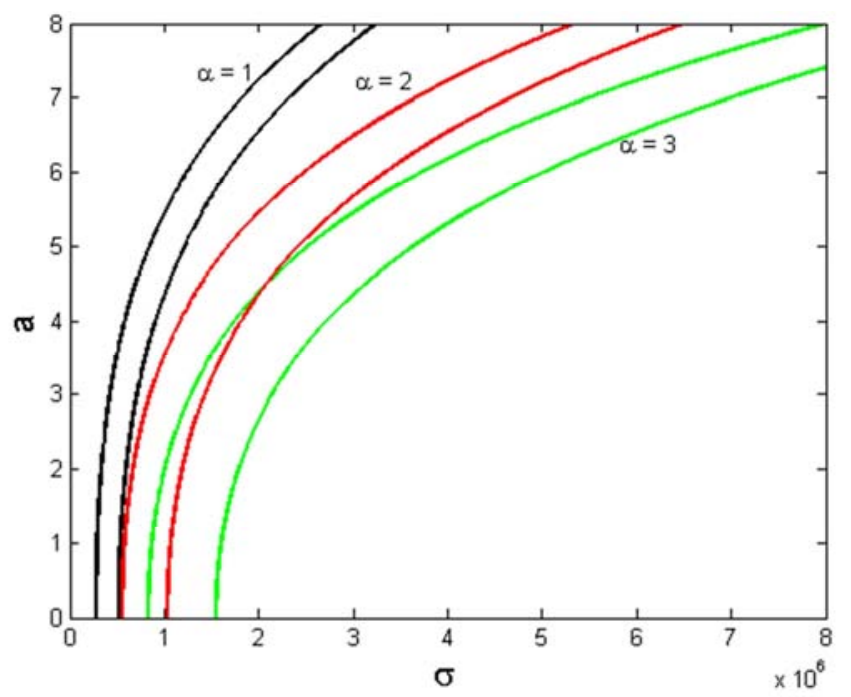

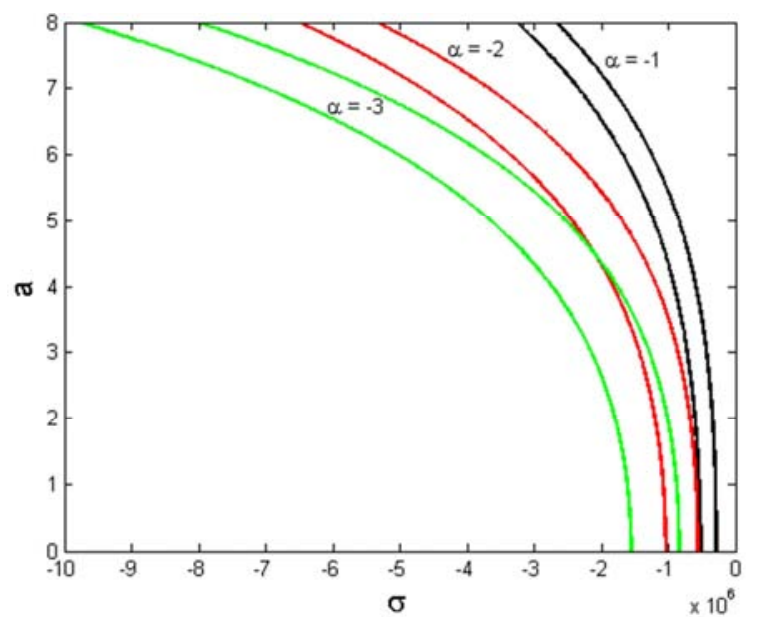

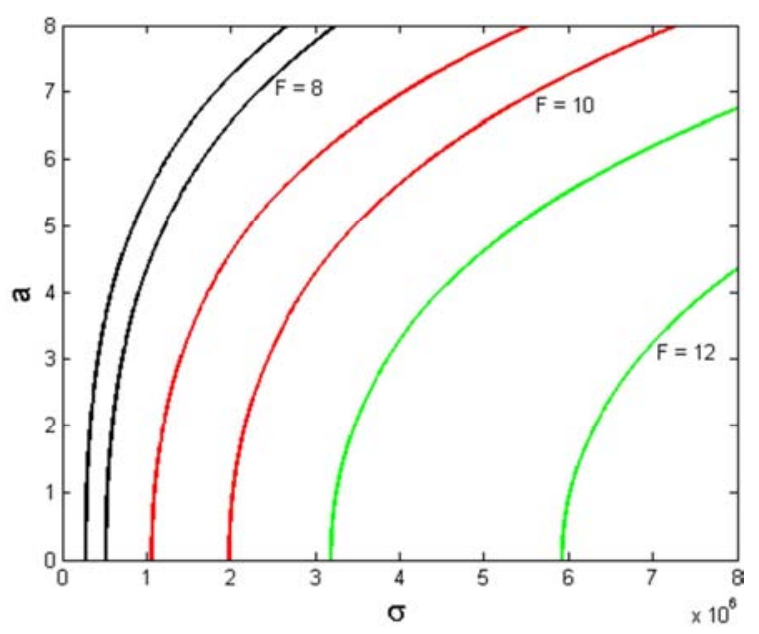

Figure 8. Frequency-response curves for super-sub-harmonic resonance of order 2 illustrating the effects of: $(a, b) \alpha$ and $(c) F$.

\section{Bifurcation and Transition to Chaos}

Since the forced damping Duffing oscillator is subjected to quintic-septic nonlinearity, then the complex phenomena must arise in the model described by (2) for different resonant states. Therefore the aim of this section is to investigate the conditions under which the complex phenomena arise in the model because they are of interest in many physics and engineering problems. For this purpose the fourth-order Runge-Kutta algorithm is used to solve numerically and draw the bifurcation diagram and its corresponding largest Lyapunov exponent of the (2). The largest Lyapunov exponent that is used as a good measure of characterization of chaos in the system is defined as follows:

$$
L y a=\lim _{t \rightarrow \infty} \frac{\ln \left(\sqrt{d u^{2}+d v_{u}^{2}}\right)}{t}
$$

Where $d u$ and $d v_{u}$ are the variations of $u$ and $u^{\prime}$ respectively. The time periodic of the periodic stroboscopic bifurcation diagram used to map the transition is $T=\frac{2 \pi}{\Omega}$. 
For set of parameters $\alpha=-0.5, \mu=0.5$ and $\Omega=1$, the bifurcation diagram and its corresponding Lyapunov exponent are represented in Figure 9. From these figures we can notice that the existence of periodic, quasiperiodic and chaotic motions. In order to have an idea on the system behavior predicted by these diagrams, various phase portraits are plotted for several different values of the excitation amplitude $F$ using the parameters of Figure 9 (see Figures 10 and 11).
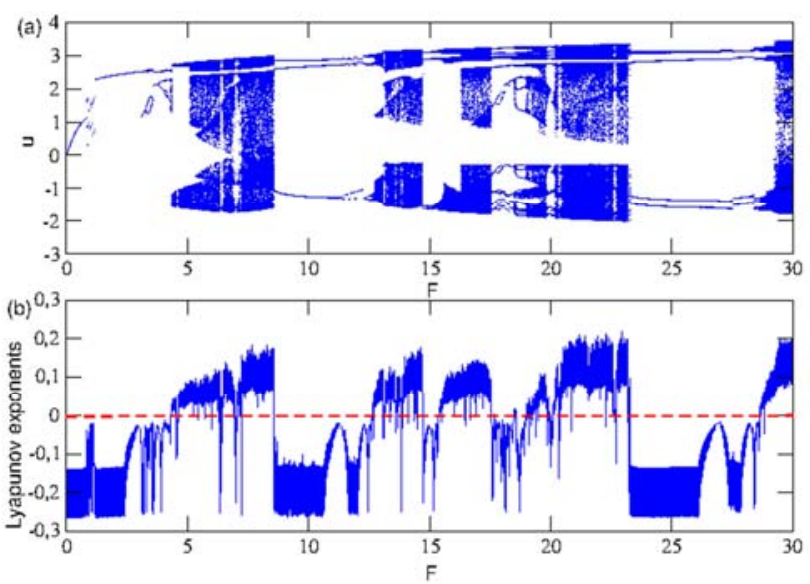

Figure 9. Bifurcation diagram and its corresponding Lyapunov of an extended Duffing oscillator versus $F$ with $\alpha=-0.5, \mu=0.5 ; \Omega=1$.
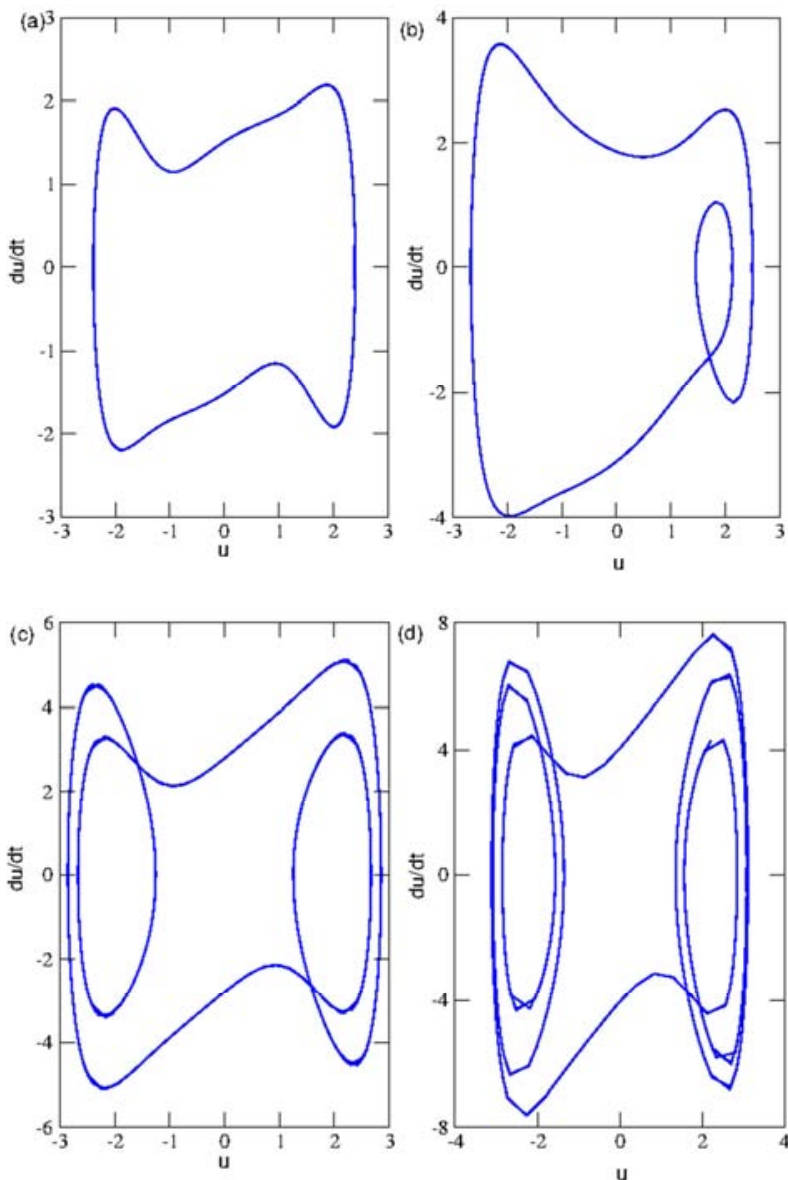

Figure 10. Periodic orbits of an extended Duffing oscillator with the parameters of Figure 9. (a): Orbit $1 T, F=2$; (b): Orbit $2 T, F=3.75$; (c): Orbit $3 T, F=10$ and (d): Orbit $4 T, F=25$.
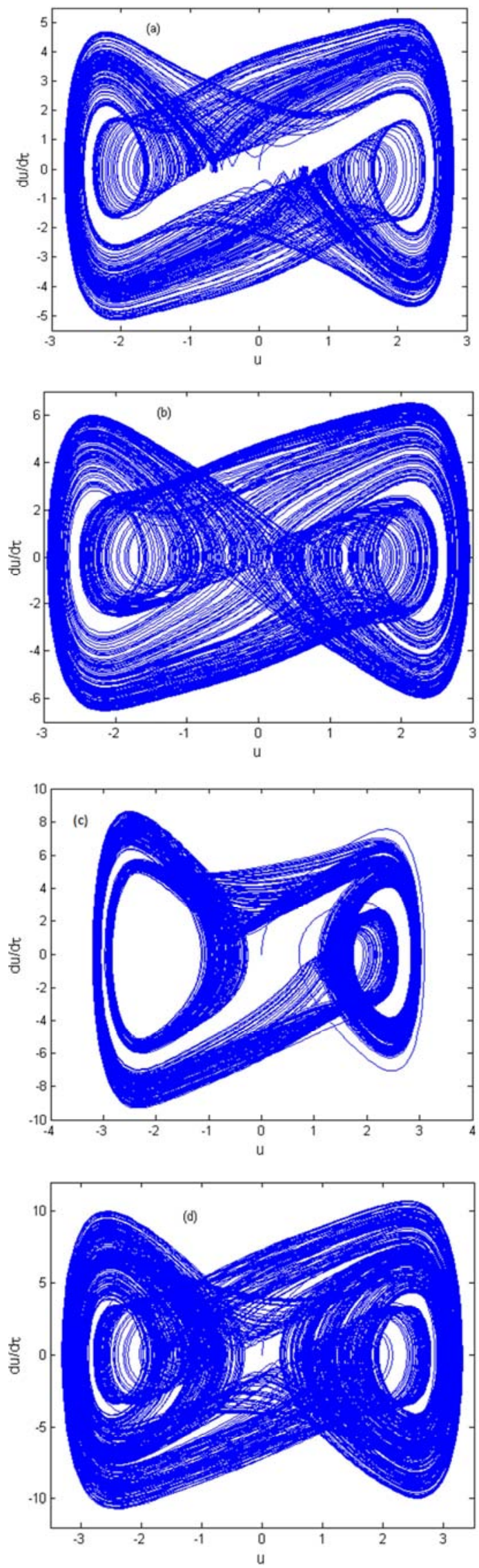

Figure 11. Chaotic orbits of an extended Duffing oscillator with the parameters of Figure 9. (a): $F=5.5$ (b): $F=8.01$; (c): $F=16.25$ and (d): $F=21.5$ 
The influence of the quintic-septic nonlinearity and linear damping coefficients on the bifurcation sequences are investigated and reported in Figures 12. From these figures it is found that decreasing of the dissipation coefficient $\mu$ accentuates the chaotic motions while increasing of quintic-septic coefficient $\alpha$ reduces the chaoticity of the system but increases the regions of the periodic oscillations. In order to detect the resonant states where the chaotic motions are dominant, the bifurcation diagrams showing the variations of $u$ versus $\Omega$ for different values of the excitation amplitude $F$ are presented in Figures13. We can note that the number of bifurcations depend on the value of $F$. Furthermore the chaotic motions are also influenced by the excitation amplitude and resonant frequency values. To illustrate such situation, the phase portraits are represented for different values of the excitation amplitude $F$ (see Figures14). We observe that for $F=8.01$ the system under study has the possibility to display chaotic motions for the primary resonance and super-sub-harmonic resonance of order 2. Moreover when $F=21.5$, the system presents in addition chaotic behavior at the sub-super-harmonic resonance of order 2 .
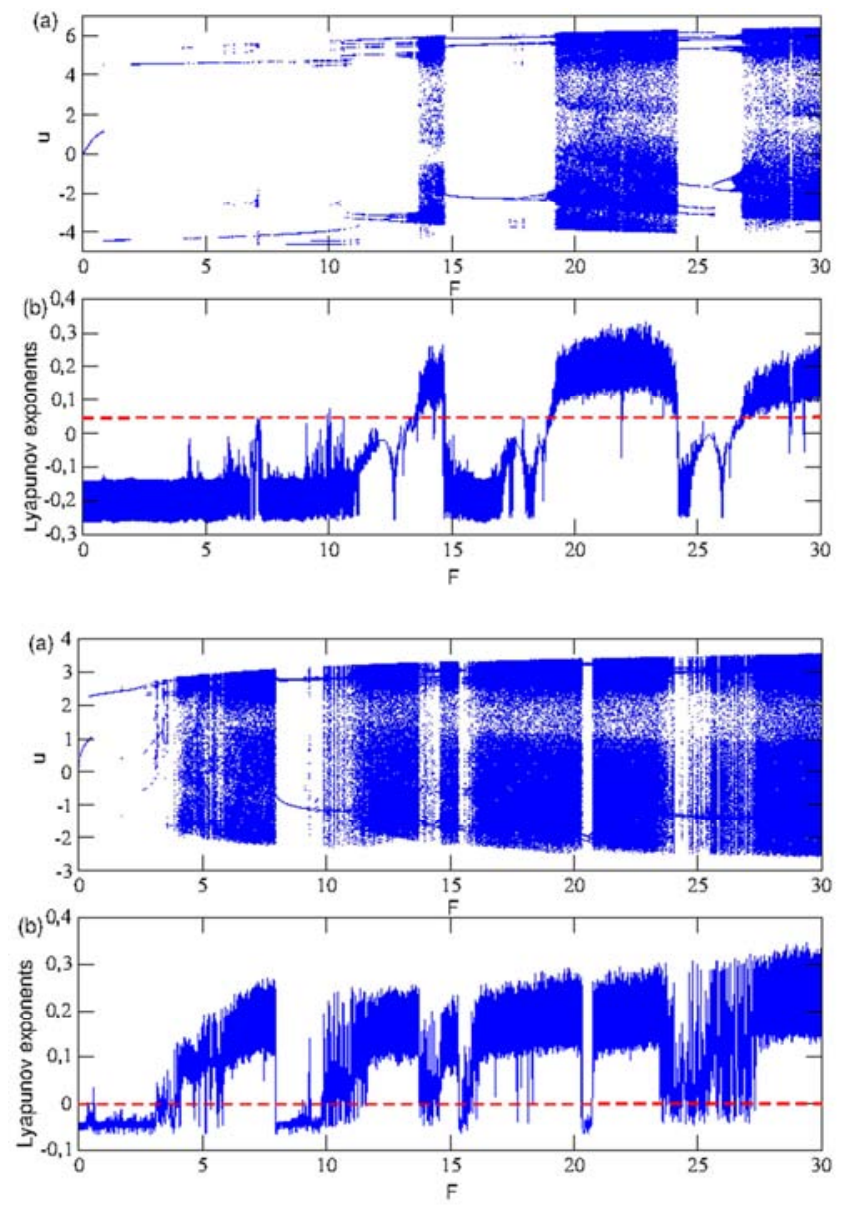

Figure 12. Effect of $\alpha=-0.01$ (left) $\mu=0.1$ (right) on the Bifurcation diagram and its corresponding Lyapunov with the parameters of Figure 9.
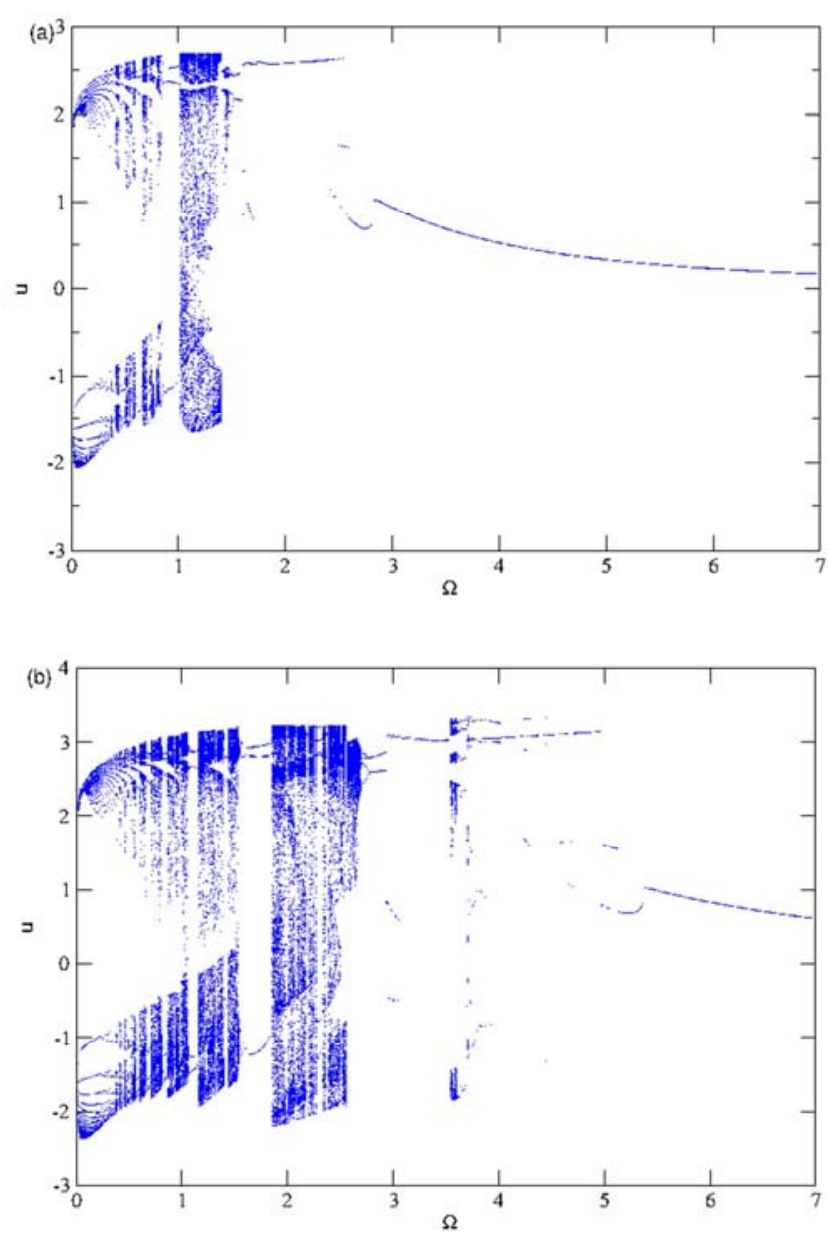

Figure 13. Bifurcation diagrams of an extended Duffing oscillator versus $\Omega$ with the parameters of Figure 9. (a): $F=8.01$ and $(b): F=29.5$.

\section{Conclusion}

In this work we have presented the study of chaotic dynamics of a cubic-quintic-setpic Duffing oscillator under periodic excitation. The particularity of this study is linked at fact that one only parameter is here used to control the quintic-septic nonlinearity. The multiple scales method applied to this extended Duffing equation has generated thirteen resonance states which of seven are thoroughly studied. The modulation equations of each considered resonance oscillation case are derived. The steady-states solutions and theirs stabilities are determined. The frequency-amplitude curves obtained show that the model displays mixed behavior, limit cycles, hysteresis, bifurcation and jump phenomena. It is found that these phenomena are considerably affected by quintic-septic nonlinearity coefficient and excitation amplitude. The numerical simulations directly performed on the model through bifurcation and Lyapunov exponent diagrams have revealed that periodic, multi-periodic oscillations and chaotic motions take place in the system. These motions predicted by these diagrams are confirmed by various phase portraits. The effects of linear dissipation $\mu$, quintic-septic nonlinearity $\alpha$ and external amplitude influence strongly the chaotic behavior of 
the system. Furthermore it is also found that the capacity of the system has developed more chaotic motions depend on the values of excitation amplitude $F$ and resonant frequency $\Omega$. Finally we can affirm that the carried on objective in this work is attained since the quintic-septic nonlinearity affects the dynamic of softening damping Duffing oscillator.
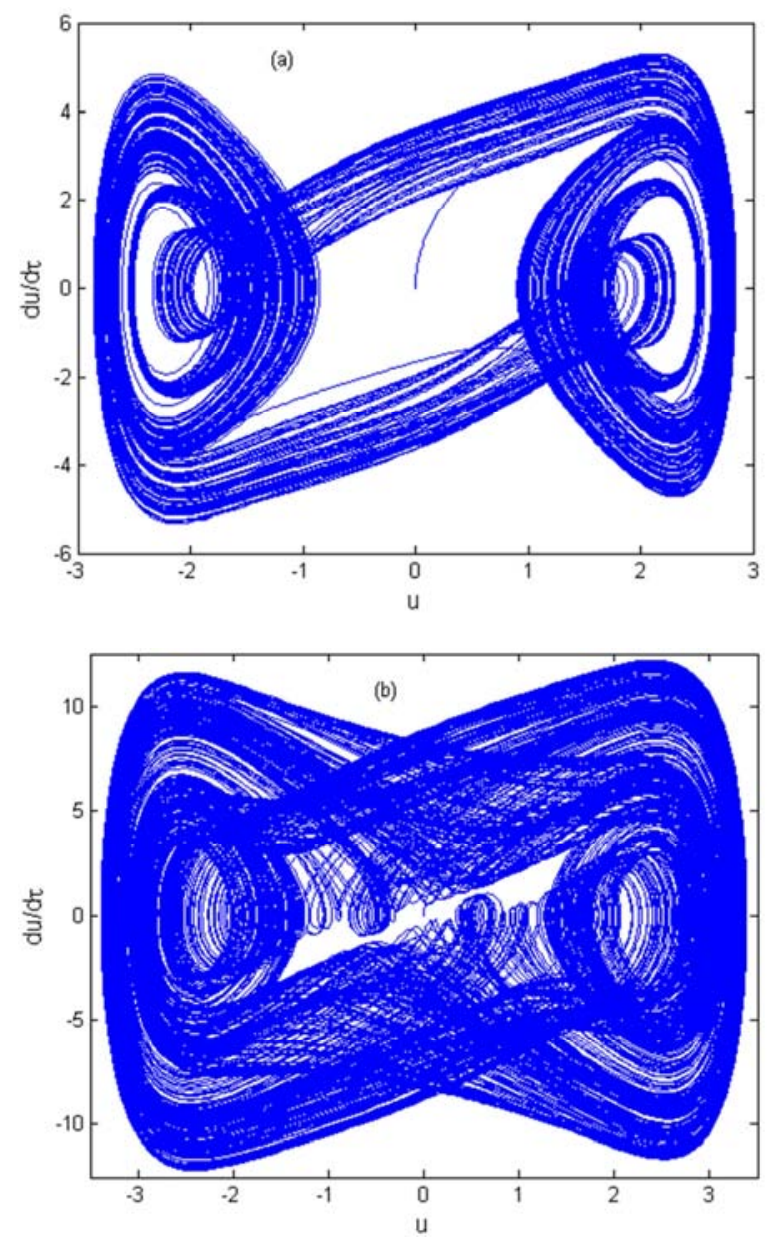

Figure 14. Phase Portraits of an extended Duffing oscillator for super-suband sub-super-harmonic resonance states with the parameters of Figure 9. (a): $F=8.01, \Omega=1 / 2$ and $(b): F=21.5, \Omega=2$.

\section{References}

[1] Monwanou, A. V., Miwadinou, C. H., Aïnamon, C., and Chabi Orou, J. B. (2018). Hysteresis, Quasiperiodicity and Chaoticity in a Nonlinear Dissipative Hybrid Oscillator, International Journal of Basic and Applied Sciences, 7(1), 1-7.

[2] Higgins, John P. (2002). Nonlinear Systems in Medicine, Yale Journal of biology and medicine, 75(5-6), 247-260.

[3] Moon, Francis C. (2004). Chaotic Vibrations An Introduction for Applied Scientists and Engineers, John Wiley.

[4] Weiss, James N., Garfinkel, Alain., Spano, Mark L., and Ditto, William L. (1994). Chaos and Chaos Control in Biology, The journal of Clinical Investigation, 93(4), 1355-1360.

[5] Tamastel, and Gruiz, Marton (2006). Chaotic Dynamics an Introduction Based on Classical Mechanics, Cambridge
University Press, New York.

[6] Hayes, Scott., Grebogi, Celso., Ott, Edward and Mark, Andrea. (1994), Experimental Control of Chaos for Communication, Physical review letters, 73(13), 1781-1784.

[7] Aihara, Kazuyuki. (2002). Chaos Engineering and Its Application to Parallel Distributed Processing With Chaotic Neural Networks using energy balance method, Proceeding of the IEEE, 90(5), 919-930.

[8] Aihara, Kazuyuki. (2012). Chaos and its applications, Procedia IUTAM, 5, 199 -203.

[9] Khan Ayub and Kumar, Sanjay. (2018). Study of chaos in chaotic satellite systems, Pramana-J. Phys, 90(13), 1-9.

[10] Mallik, A. K. (2003). Response of A Hard Duffing Oscillator to Harmonic Excitation-An Overview, indian institute of technology, kharagpur 721302, 28-30, 1-5.

[11] Luo, Albert C. J., and Huang Jianzhe. (2014). Period-3 Motions to Chaos in a Softening Duffing Oscillator, International Journal of Bifurcation and Chaos, 24(3), 1430010-1430010-26

[12] Ghandchi-Tehrani Maryam., Wilmshurst, Lawrence I., and Stephen, J. Elliote. (2015). Bifurcation control of a Duffing oscillator using pole placement, Journal of Vibration and Control, 21(14), 2838-2851.

[13] Kovacic, Ivanaand Brennan, Michael J. (2011). The Duffing Equation Nonlinear Oscillators and their Behaviour, John Wiley \& Sons, Ltd.

[14] Lou, Jing-jun., He, Qi-wei., and Zhu Shi-jian. (2004). Chaos In The Softening Duffing System Under Multi-Frequency Periodic Forces, Applied Mathematics and Mechanics, 25(12), 1421-1427.

[15] Berger, J. E., and Nunes, G. Jr. (1997). A mechanical Duffing oscillator for the undergraduate laboratory, American Journal of Physics, 65(9), 841-846.

[16] Nayfeh, A. H. and D. T. Mook. (1995). Nonlinear Oscillations, John Wiley \& Sons, New York.

[17] Donso, Guillermo and Celso L. Ladera. (2012). Nonlinear dynamics of a magnetically driven Duffing-type spring-magnet oscillator in the static magnetic field of a coil, European Journal of Physics, 33(6), 1473-1486.

[18] Chua, Vivien. (2012). Cubic-Quintic Duffing Oscillators, www.its.caltech.edu/mason/research/duf.pdf, 1-19.

[19] Oyesanya, Moses O., and Nwamba, J. I. (2013). Stability analysis of damped cubic-quintic Duffing oscillator, WorldJournal of Mechanics, 3(1), 43-57.

[20] Kacem, N., Baguet, S., Dufour R., and Hentz, S. (2011). Stability control of nonlinear micromachanical resonators under simultaneous primary and superharmonic resonances, Applied Physics Letters, 98(19), 193507-193507-3.

[21] Kacem, N., and Hentz, S. (2009) Bifurcation topology tuning of a mixed behavior in nonlinear micromechanical resonators, Applied PhysicsLetters, 95(18), 183104-183104-3.

[22] Elshurafa, Amro M., Khirallah, Kareem, Tawfik, Hani H., Ahmed, Emira., Ahmed K. S. Abdel Aziz and Sedky, Sherif. M. (2011). Nonlinear Dynamics of Spring Softening and Hardening in Folded-MEMS Comb Drive Resonators, Journal of Microelectromechanical Systems, 20(4), 943-958. 
[23] Oyesanya, M. O., and Nwamba, J. I. (2013). Duffing Oscillator with Heptic Nonlinearity under single Periodic Forcing, Int. J. of Mechanics and Applications, 3(2), 35-43.

[24] Lesage, J. C., Liu, M. C. (2008). On the investigation of a restrained cargo system modeled as a Duffing oscillator of various orders, Proceeding of Early Career Technical Conference, ASME, Maimi, Florida, USA.

[25] Koudahoun, L. H., Kpomahou, Y. J. F., Adjaï, D. K. K., Akande, J., Rath, B. Mallick, P. and Monsia, D. M. (2016). Periodic Solutions for nonlinear oscillations in Elastic Structures via Energy Balance Method, viXra 16110214v1, $1-12$.

[26] Deleanu, Dumitru. (2016). Transient and Steady-State
Responses for the Ship Rolling Motion with Multiple scales Lindstedt Poincaré Method, "Mircea Cel Batran" Naval Academy Scientific Bulletin, XIX(2), 208-215.

[27] Luo, Albert C. J. (2010). Dynamical Systems: Discontinuity, Stochasticity and Time-Delay, Springer-Verlag, New York.

[28] Marinca, V. and Herisanu, N. (2005). Forced Duffing Oscillator with Slight Viscous Damping and Hardening Non-Linearity, Facta Universitatis, Series: Mechanics, Automatic Control and Robotics, 4(17), 245-255.

[29] Nayfeh, Ali H. (2004). Perturbation Methods, WILEY-VCH.

[30] Hayashi, Chihiro. (1986). Nonlinear Oscillations in Physical Systems, Princeton University Press, 41 William Sheet, Princeton, New Jersey 08540. Mc Graw-Hill, Inc. 